\title{
Origin of the magnetic-field dependence of the nuclear spin-lattice relaxation in iron
}

\author{
G. Seewald, E. Zech, and H.-J. Körner \\ Physik-Department, Technische Universität München, D-85748 Garching, Germany \\ D. Borgmann \\ Institut für Physikalische und Theoretische Chemie, Universität Erlangen-Nürnberg, D-91058 Erlangen, Germany \\ M. Dietrich \\ Technische Physik, Universität des Saarlandes, D-66041 Saarbrücken, Germany \\ ISOLDE Collaboration \\ CERN, CH-1211 Geneva 23, Switzerland
}

(Received 6 December 2006; revised manuscript received 23 January 2008; published 24 March 2008)

\begin{abstract}
The magnetic-field dependence of the nuclear spin-lattice relaxation at Ir impurities in Fe was measured for fields between 0 and $2 \mathrm{~T}$ parallel to the [100] direction. The reliability of the applied technique of nuclear magnetic resonance on oriented nuclei was demonstrated by measurements at different radio-frequency (rf) field strengths. The interpretation of the relaxation curves, which used transition rates to describe the excitation of the nuclear spins by a frequency-modulated rf field, was confirmed by model calculations. The magneticfield dependence of the so-called enhancement factor for rf fields, which is closely related to the magnetic-field dependence of the spin-lattice relaxation, was also measured. For several magnetic-field-dependent relaxation mechanisms, the form and the magnitude of the field dependence were derived. Only the relaxation via eddy-current damping and Gilbert damping could explain the observed field dependence. Using reasonable values of the damping parameters, the field dependence could perfectly be described. This relaxation mechanism is, therefore, identified as the origin of the magnetic-field dependence of the spin-lattice relaxation in $\mathrm{Fe}$. The detailed theory, as well as an approximate expression, is derived, and the dependences on the wave vector, the resonance frequency, the conductivity, the temperature, and the surface conditions are discussed. The theory is related to previous attempts to understand the field dependence of the relaxation, and it is used to reinterpret previous relaxation experiments in Fe. Moreover, it is predicted that the field dependences of the relaxation in $\mathrm{Fe}$ and $\mathrm{Co}$, on one side, and in $\mathrm{Ni}$, on the other side, differ substantially, and it is suggested that the literature values of the high-field limits of the relaxation constants in Fe are slightly too large.
\end{abstract}

DOI: 10.1103/PhysRevB.77.104433

PACS number(s): 76.60.Es, 75.50.Bb, 75.30.Ds, 76.80.+y

\section{INTRODUCTION}

The magnetic-field dependence of the nuclear spin-lattice relaxation in $\mathrm{Fe}, \mathrm{Co}$, and $\mathrm{Ni}$ had been an unsolved problem for more than 30 years. ${ }^{1-3}$ The effect typically manifests itself at low applied magnetic fields by relaxation rates that are 2-10 times larger than in the high-field limit, which is essentially reached within applied fields of the order of $1 \mathrm{~T}$. Since there is a close relation between the spin-lattice relaxation and low-frequency magnetic-moment fluctuations, ${ }^{4,5}$ the lack of an explanation would point to a fundamental deficiency in our understanding of the moment fluctuations in $\mathrm{Fe}, \mathrm{Co}$, and $\mathrm{Ni}$. This was the motivation to obtain more information on the effect.

A phenomenological description of the effect had been proposed by Kopp and Klein: According to their enhancement factor model (EFM), the field-dependent part of the spin-lattice relaxation is proportional to the square of the NMR enhancement factor. ${ }^{6}$ In this way, the magnetic-field dependence of the relaxation is attributed to the magneticfield dependence of the enhancement factor. The EFM provided a description of the field dependence of the relaxation in polycrystalline samples, ${ }^{6,7}$ and it was consistent with the main features of the field dependence in single-crystal samples, in particular, with the occurrence of peaks for certain directions of the magnetic field. ${ }^{2}$

However, a critical experimental test of the EFM was still missing, because in polycrystalline samples, the field dependence of the enhancement factor is not well known, and the few relaxation experiments on single-crystal samples ${ }^{8,9}$ had not been interpreted quantitatively by the EFM. In this work, a single-crystal sample was used and the magnetic field was applied along the [100] direction. The field dependence of the enhancement factor is well known for that geometry. Moreover, it was also determined experimentally. This enabled us to establish the actual relationship between the relaxation and the enhancement factor. It turned out to differ from the postulated quadratic dependence on the enhancement factor.

In context with the field dependence of the spin-lattice relaxation in $\mathrm{Fe}, \mathrm{Co}$, and $\mathrm{Ni}$, several relaxation mechanisms had been discussed, but none of those could explain the effect. ${ }^{1,8,10-12}$ It had been speculated that this failure might not be due to the inadequacy of the proposed relaxation mechanisms, but due to an incomplete knowledge of the magnetization behavior, the band structure, or the spin-wave dispersion. $9,13,14$ The precise data and the close examination of those mechanisms in this work show, however, that those speculations are not true. In contrast, it turns out that an 
important mechanism has been ignored so far, the relaxation via eddy currents and Gilbert damping. The theory of this relaxation mechanism is derived and it is shown that it can explain the observed field dependence.

Since the reliability of relaxation measurements by nuclear magnetic resonance on oriented nuclei (NMR-ON), the technique that was used in this work, had been questioned in the past, ${ }^{3,7,14}$ the theory of NMR-ON was also reexamined. In particular, the practice to use transition rates to describe the effect of a coherent rf field on the sublevel populations had been doubted. Therefore, model calculations that showed under which conditions that practice is justified were performed. In addition, the reliability of the technique was tested by measurements at different rf-field strengths.

The relaxation measurements were performed on radioactive ${ }^{186} \mathrm{Ir}$ and ${ }^{189} \mathrm{Ir}$ nuclei, which were coimplanted into the Fe sample. One part of the experiment, the determination of the electric-quadrupolar contribution to the relaxation by the comparison of the relaxations of both isotopes, was already treated in Ref. 15. The present work is mainly concerned with the form and the magnitude of the field dependence of the relaxation, which were deduced from the data on ${ }^{186} \mathrm{Ir}$.

\section{ENHANCEMENT FACTOR}

The NMR enhancement factor $\eta$ in ferromagnets is defined as the ratio of the effective rf magnetic field at the nuclear site to the applied rf field. ${ }^{16}$ It takes into account that the magnetization and the hyperfine field are slightly displaced toward the instantaneous direction of the rf field. The resulting transverse component of the hyperfine field acts at the nuclear site as an additional rf field, which is much larger than the applied rf field. It can be shown that

$$
\eta=1+\frac{B_{\mathrm{HF}}}{B_{\eta}} \approx \frac{B_{\mathrm{HF}}}{B_{\eta}},
$$

where $B_{\mathrm{HF}}$ is the hyperfine field and $B_{\eta}$ is the effective field that holds the magnetization in its equilibrium position. An appropriate expression to calculate $B_{\eta}$ as a function of the applied magnetic field $B_{\text {ext }}$ is given in Refs. 8 and 17 .

Within the EFM of Ref. 6, the relaxation rate $R$ is the sum of a high-field limit and a field-dependent contribution that is proportional to $\eta^{2}$. To increase the flexibility of the model, we assume that the latter contribution is proportional to $\eta^{\xi}$, where the exponent $\xi$ is not necessarily 2 :

$$
R\left(B_{\mathrm{ext}}\right)=R(\infty)+[R(0)-R(\infty)]\left[\frac{\eta\left(B_{\mathrm{ext}}\right)}{\eta(0)}\right]^{\xi} .
$$

The original idea behind the EFM was that the internal fields that are responsible for the field-dependent part of the relaxation are similarly enhanced as the rf field. The weak point of that idea was that those internal fields had never been specified. Nevertheless, it makes sense to try to describe the field dependence in terms of $\eta$, since $\eta$ can be viewed just as a synonym of $B_{\eta}^{-1}$. In this sense, $\eta$ is relevant for the long-wavelength magnetic excitations of the system in several ways: For example, $\eta$ is essentially equivalent to the transversal susceptibility, which describes the displace- ment of the magnetization in response to forces that act on the magnetization as a whole. However, $\eta$ is also inversely proportional to the lowest frequency of the spin-wave spectrum.

In this work, the magnetic field was applied along the [001] direction in the (110) plane of a Fe single-crystal disk. The rf field was also applied within the sample plane. For that geometry, $B_{\eta}$ is well known:

$$
\begin{gathered}
B_{\eta}=B_{a} \text { for } B_{\mathrm{ext}} \leqslant B_{\mathrm{dem}}^{(0)}, \\
B_{\eta}=B_{a}+B_{\mathrm{ext}}-B_{\mathrm{dem}}^{(0)} \text { for } B_{\mathrm{ext}}>B_{\mathrm{dem}}^{(0)} .
\end{gathered}
$$

Here, $B_{a}$ is the anisotropy field $(0.059 \mathrm{~T}$ in $\mathrm{Fe})$ and $B_{\mathrm{dem}}^{(0)}$ is the magnitude of the demagnetization field for the fully magnetized sample.

The independence from $B_{\text {ext }}$ for $B_{\text {ext }} \leqslant B_{\text {dem }}^{(0)}$ is due to the shielding of $B_{\text {ext }}$ by the demagnetization field: The shielding is complete during the magnetization of the sample when the domains with the magnetization parallel to $\mathbf{B}_{\text {ext }}$ grow at the expense of the other domains. The magnetization of the sample is completed at $B_{\text {ext }}=B_{\text {dem }}^{(0)}$, which thus marks the transition from the multidomain to the one-domain regime.

Two features of Eqs. (1) and (3) deserve special attention. First, the frequency dependence of $\eta$ is neglected, because the relevant electronic resonance frequency, which is of the order of

$$
\left(\gamma_{e} / 2 \pi\right)\left(B_{\eta} 4 \pi M\right)^{1 / 2} \geqslant 10.6 \mathrm{GHz},
$$

is much larger than the frequencies applied in this work. Second, to obtain the correct dependence on $B_{\mathrm{dem}}^{(0)}$, it must be taken into account that, due to the skin effect, the magnetization $\mathbf{M}$ is displaced by the rf field only in a very thin surface layer. The demagnetization field in that layer is not displaced, since it originates largely from the rest of the sample. Therefore, the demagnetization field of the undisturbed sample acts on $\mathbf{M}$ of the surface layer like an external field. This gives in the end the dependence on $B_{\mathrm{dem}}^{(0)}$ of Eq. (3).

\section{FIELD-DEPENDENT RELAXATION MECHANISMS}

Spin-lattice relaxation rates in metals are specified by the reciprocal value of the Korringa constant, $R=\left(T_{1} T\right)^{-1}$. Usually, the dominant relaxation mechanism is the scattering of conduction electrons via the hyperfine interaction at the nuclear site, ${ }^{18,19}$ and $R$ is magnetic-field independent, because the involved matrix elements and densities of states are practically field independent. In this section, we discuss several mechanisms by which the ferromagnetism can introduce a field dependence. They have in common that they arise from the coupling of the nuclear spin to the magnetization vector. Since, in this case, the susceptibility formalism proves to be convenient, it is discussed first.

\section{A. Susceptibility formalism}

\section{Formalism}

Within the susceptibility formalism, $, 411,20$ 


$$
R=\frac{k_{B}}{\hbar} \frac{K^{2}}{\hbar \omega_{n}\left(\hbar \gamma_{e}\right)^{2}} \frac{2 V}{(2 \pi)^{3}} \int \operatorname{Im}\left[\chi\left(\mathbf{q}, \omega_{n}\right)\right] d^{3} q .
$$

Here, $K$ is the coupling constant between the nuclear and the electronic spins, $\chi(\mathbf{q}, \omega)$ is the transversal dynamical susceptibility in units of the induced magnetic moment per atom and magnetic-field unit, $V$ is the volume per atom, $\gamma_{e}$ is the gyromagnetic ratio of the electron spins, and $\omega_{n}$ is the nuclear precession frequency.

The magnitude of $\omega_{n}$ is $2 \pi \nu_{n}$ and the sign is given by

$$
\operatorname{sgn}\left(\omega_{n}\right)=-\operatorname{sgn}\left(B_{\mathrm{HF}}\right) \operatorname{sgn}\left(\gamma_{n}\right),
$$

whereas the nuclear resonance frequency $\nu_{n}$ is defined in this work as a positive quantity. $\gamma_{n}$ is the nuclear gyromagnetic ratio. In connection with the sign of $\omega_{n}$, it should be noted that the decisive sign is

$$
\operatorname{sgn}\left(\omega_{n} \gamma_{e}\right)=-\operatorname{sgn}\left(B_{\mathrm{HF}}\right) \operatorname{sgn}\left(\gamma_{n}\right) \operatorname{sgn}\left(\gamma_{e}\right),
$$

which is negative if the nuclear and the electronic spins precess in the same sense and positive if they precess in the opposite sense.

If several different coupling constants and electronic magnetic moments contribute to the spin-lattice relaxation, each contribution is given by an expression of the form of Eq. (4). In this section, almost exclusively the contribution from the coupling to the magnetization vector via the static hyperfine interaction is discussed. $R_{\mathrm{sw}}$ denotes the respective contribution to the relaxation constant. The coupling constant in the case of $R_{\mathrm{sw}}$ is the static hyperfine coupling constant ${ }^{11,21,22}$

$$
K=\left(\hbar \omega_{\mathrm{HF}}\right) / S \approx\left(\hbar \omega_{n}\right) / S,
$$

where $S=(M V) /\left(\hbar\left|\gamma_{e}\right|\right)$ is the electronic spin and $\omega_{\mathrm{HF}}$ is the precession frequency due to the static hyperfine field. For simplicity, in this context, $\omega_{\mathrm{HF}}$ is approximated by $\omega_{n}$, assuming $B_{\mathrm{HF}} \gg B_{\text {ext }}$. This is a good approximation for ferromagnetic transition metals, where the hyperfine fields are of the order of 10-100 T. The susceptibility in the case of $R_{\mathrm{sw}}$,

$$
\chi(\mathbf{q}, \omega)=\hbar\left|\gamma_{e}\right| S \frac{1}{2}\left(\frac{\partial}{\partial b_{x}^{\prime}}+i \frac{\partial}{\partial b_{y}^{\prime}}\right) \frac{m_{x}-i m_{y}}{M},
$$

describes the displacement of the magnetization in response to a small, complex, space- and time-periodic, transversal field $\mathbf{b}^{\prime}$ that is proportional to $\exp (i \mathbf{q r}-i \omega t)$. Here, $m_{x}$ and $m_{y}$ are the transversal components of the displaced magnetization, which are also proportional to $\exp (i \mathbf{q r}-i \omega t)$.

$\chi$ is obtained from the linearized equation of motion of $\mathbf{m}$, which turns out to be of the form

$$
\begin{gathered}
\frac{d}{d t} \frac{m_{x}}{M}=+\omega_{x} \frac{m_{y}}{M}-\gamma_{e} b_{y}^{\prime}, \\
\frac{d}{d t} \frac{m_{y}}{M}=-\omega_{y} \frac{m_{x}}{M}+\gamma_{e} b_{x}^{\prime} .
\end{gathered}
$$

This equation has the solution

$$
\frac{m_{x}}{M}=\frac{\omega_{x} \gamma_{e} b_{x}^{\prime}+i \omega \gamma_{e} b_{y}^{\prime}}{\omega_{x} \omega_{y}-\omega^{2}},
$$

$$
\frac{m_{y}}{M}=\frac{-i \omega \gamma_{e} b_{x}^{\prime}+\omega_{y} \gamma_{e} b_{y}^{\prime}}{\omega_{x} \omega_{y}-\omega^{2}} .
$$

Combining Eqs. (4), (7), (8), and (10), one obtains

$$
R_{\mathrm{sw}}=\frac{k_{B} \omega_{n} V}{\hbar S(2 \pi)^{3}} \operatorname{sgn}\left(\gamma_{e}\right) \int \operatorname{Im}\left[\frac{\omega_{x}+\omega_{y}-2 \omega_{n}}{\omega_{x} \omega_{y}-\omega_{n}^{2}}\right] d^{3} q,
$$

where $\omega_{x}$ and $\omega_{y}$ are functions of $\mathbf{q}$ and $\omega=\omega_{n}$.

In this way, the susceptibility formalism relates all relaxation mechanisms that arise from the coupling to the magnetization vector to the equation of motion of the magnetization. Note that this equation is naturally closely related to the spin-wave spectrum, since displacements of the magnetization that are proportional to $\exp (i \mathbf{q r}-i \omega t)$ are just spin waves. The problem is now to find the equation of motion.

\section{Equation of motion of the magnetization}

The magnetization precesses around an effective field that is the sum of the magnetic field $\mathbf{B}$, the anisotropy field $\mathbf{B}_{a}$, the exchange field, internal fields $\mathbf{b}^{(j)}$ due to the coupling to other excitation modes, and $\mathbf{b}^{\prime}$ :

$$
\frac{d}{d t} \mathbf{M}=\gamma_{e} \mathbf{M} \times\left(\mathbf{B}+\mathbf{B}_{a}+\frac{D \Delta \mathbf{M}}{\hbar\left|\gamma_{e}\right| M}+\sum_{j} \mathbf{b}^{(j)}+\mathbf{b}^{\prime}\right),
$$

where $D$ is the spin-wave stiffness constant. This equation must be solved together with Maxwell's equations and the equations of motion of the other excitation modes.

If the two explicitly time-dependent Maxwell equations are combined and the displacement current is neglected, one obtains

$$
-\Delta \mathbf{B}+\frac{4 \pi \sigma}{c^{2}} \frac{d}{d t} \mathbf{B}=4 \pi[-\Delta \mathbf{M}+\nabla(\nabla \mathbf{M})],
$$

where $\sigma$ is the conductivity. Since $\mathbf{b}^{\prime}$ describes the hyperfine interaction acting on the electron spin, it is not a "true" magnetic field and does not appear in Maxwell's equations. To linearize the equation of motion, $\mathbf{M}$ and $\mathbf{B}$ are decomposed into large, static, and uniform $z$ components and small transversal components $\mathbf{m}$ and $\mathbf{b}$, which are proportional to $\exp (i \mathbf{q r}-i \omega t)$. The longitudinal components are approximately given by

$$
M_{z}=M, \quad\left(B+B_{a}\right)_{z}=B_{\eta}+4 \pi M .
$$

b and $\mathbf{m}$ are related by Eq. (13). Making use of the periodicities of those quantities, one obtains

$$
\begin{aligned}
& b_{x}=4 \pi m_{x} \frac{q^{2} \delta^{2} \cos ^{2} \theta}{q^{2} \delta^{2}-2 i \operatorname{sgn}(\omega)}, \\
& b_{y}=4 \pi m_{y} \frac{q^{2} \delta^{2}}{q^{2} \delta^{2}-2 i \operatorname{sgn}(\omega)},
\end{aligned}
$$

where $\delta$ is the skin depth: 


$$
\delta=\frac{c}{(2 \pi \sigma|\omega|)^{1 / 2}} .
$$

$\theta$ is the angle between $\mathbf{q}$ and the direction of the magnetization, and $x$ denotes the transversal component parallel to $\mathbf{q}$, which gives

$$
q_{x}=q \sin \theta, \quad q_{y}=0, \quad q_{z}=q \cos \theta .
$$

If Eqs. (12), (14), and (15) are combined, and if only terms of first order in $\mathbf{m}$ are retained, one obtains the linearized equation of motion (9) with the parameters

$$
\begin{aligned}
& \omega_{x}=\omega_{x}^{(0)}+\sum_{j} \omega_{x}^{(j)}, \\
& \omega_{y}=\omega_{y}^{(0)}+\sum_{j} \omega_{y}^{(j)} .
\end{aligned}
$$

Here,

$$
\begin{gathered}
\omega_{x}^{(0)}=\gamma_{e}\left(B_{\eta}+\frac{D q^{2}}{\left|\gamma_{e}\right| \hbar}\right), \\
\omega_{y}^{(0)}=\gamma_{e}\left(B_{\eta}+4 \pi M \sin ^{2} \theta+\frac{D q^{2}}{\left|\gamma_{e}\right| \hbar}\right)
\end{gathered}
$$

are the parameters without eddy-current damping and coupling to other excitation modes. One set of $\omega^{(j)}$ 's,

$$
\begin{aligned}
& \omega_{x}^{(\mathrm{ed})}=\gamma_{e} 4 \pi M \frac{2}{2+i \operatorname{sgn}(\omega) \delta^{2} q^{2}}, \\
& \omega_{y}^{(\mathrm{ed})}=\gamma_{e} 4 \pi M \frac{2(\cos \theta)^{2}}{2+i \operatorname{sgn}(\omega) \delta^{2} q^{2}},
\end{aligned}
$$

are the contributions from the eddy-current damping. The other $\omega^{(j)}$ 's are related to the internal fields from other excitation modes by

$$
\begin{aligned}
& \omega_{x}^{(j)}=-\gamma_{e} \frac{M}{m_{y}} b_{y}^{(j)}, \\
& \omega_{y}^{(j)}=-\gamma_{e} \frac{M}{m_{x}} b_{x}^{(j)} .
\end{aligned}
$$

In $\mathrm{Fe}, \quad D=280 \mathrm{meV}^{2}, \quad \gamma_{e}=184 \mathrm{GHz} \mathrm{T}^{-1}, \quad 4 \pi M$ $=2.219 \mathrm{~T}, V=11.7 \AA^{3}$, and $S=1.06 .{ }^{23}$ The expressions for $\omega_{x}^{(0)}$ and $\omega_{y}^{(0)}$ are well known from treatments of the spectrum of the spin-wave resonance frequencies, ${ }^{24-26}$ which are given by

$$
\frac{\left(\omega_{x}^{(0)} \omega_{y}^{(0)}\right)^{1 / 2}}{2 \pi} .
$$

Note the dependence of $\omega_{x}^{(0)}$ and $\omega_{y}^{(0)}$ on $B_{\eta}$. It is the source of the field dependence of the spin-lattice relaxation for all the relaxation mechanisms that are discussed below. Also note that, in general, $\omega_{x}^{(0)} \neq \omega_{y}^{(0)}$ due to the demagnetization fields of the spin waves in the $x$ direction. As a result, the precession of the magnetization is, in general, elliptic.

The expressions for $\omega_{x}^{(\mathrm{ed})}$ and $\omega_{y}^{(\mathrm{ed})}$ should be complemented by the $q$ dependence of $\delta$, since $\sigma$ and $\delta$ become $q$ dependent, when the wavelength becomes shorter than the mean free path $\Lambda$ of the conduction electrons. $\sigma$ and $\delta$ are given in terms of the normal conductivity $\sigma_{0}$ and the normal skin depth $\delta_{0}$, which represent the limit $\Lambda q \ll 1$, by the following relation, which is well known from treatments of the anomalous skin effect: ${ }^{27,28}$

$$
\frac{\sigma}{\sigma_{0}}=\frac{\delta_{0}^{2}}{\delta^{2}}=\frac{3}{2(\Lambda q)^{2}}\left\{\left[1+(\Lambda q)^{2}\right] \frac{\arctan (\Lambda q)}{\Lambda q}-1\right\} .
$$

At this point, it is also useful to introduce the length scales $\delta_{m}$ and $l_{d} . \delta_{m}^{-1}$ is defined as that $q$ that fulfills the relation

$$
q \delta=\left(\frac{8 \pi M}{B_{\eta}}\right)^{1 / 2}
$$

$\delta_{m}$ can be interpreted as an effective rf penetration depth that takes the magnetic permeability into account. For $q \delta_{m} \gg 1$, the eddy-current term is a small modification of the equation of motion of the magnetization; for $q \delta_{m} \leqslant 1$, it dominates that equation.

$$
l_{d}=\left(\frac{D}{\hbar\left|\gamma_{e}\right| B_{\eta}}\right)^{1 / 2}
$$

is a typical length scale of spatial variations of the direction of the magnetization, as they occur, for example, at domain walls. $\omega_{x}^{(0)}$ and $\omega_{y}^{(0)}$ are independent of $q$ for $q l_{d} \ll 1$ and are proportional to $q^{2}$ for $q l_{d} \gg 1$. Usually, $l_{d} \ll \delta_{m}$. For example, for $B_{\eta}=0.059 \mathrm{~T}$ and the parameters that are used in this work to describe the relaxation of ${ }^{186} \mathrm{IrFe}$, typical numbers are $\delta_{m}=0.14 \mu \mathrm{m}$ and $l_{d}=0.020 \mu \mathrm{m}$.

\section{Virtual excitation of spin waves}

If $\nu_{n}$ lies within the spin wave resonance spectrum, the nuclear spins can emit and absorb spin waves. This relaxation mechanism is discussed in Sec. III B. In contrast, if $\nu_{n}$ is smaller than the lowest spin-wave resonance frequency, only a virtual excitation of spin waves takes place, which can be viewed as a dynamic displacement of the magnetization in the vicinity of the nuclear spin or as an admixture of spin waves to the magnetic sublevels of the nuclear spin. It contributes to the relaxation, if the virtually excited spin waves decay to some other excitation mode that can be excited at $\nu_{n}$. This relaxation mechanism can be viewed in different ways: (i) It can be viewed as an excitation of the final excitation mode, where the virtual excitation of spin waves acts as an additional, indirect coupling between the nuclear spins and that mode. (ii) It can be viewed as an excitation of spin waves, where the spin-wave resonance spectra are broadened by the decay of the spin waves to the final excitation mode so that the tails of the spectra extend down to $\nu_{n}$. (iii) Within the susceptibility formalism, the equation of motion of the magnetization is modified by the coupling of the spin waves to the final excitation mode in such a way that the imaginary part of the susceptibility no longer vanishes at $\nu_{n}$.

Since there are several decay modes of the spin waves, several relaxation mechanisms via the virtual excitation of spin waves can be distinguished. To find potentially relevant 
decay modes, one can proceed in different ways: In previous work, it was looked for excitations that couple to the spin waves and that have a resonance spectrum that extends down to zero. The obvious elementary excitations in this context are sound waves and various single electron-hole excitations. The relaxation via the indirect coupling to those excitations is discussed in Secs. III C and III D, respectively. As an alternative approach, in this work, we also consider the equation of motion that is commonly used to describe the ferromagnetic resonance. That equation contains two damping terms, the eddy-current and the Gilbert damping. The relaxation via those terms is discussed in Secs. III E and III F, respectively.

\section{Approximations}

The integrand of Eq. (11),

$$
F=\operatorname{Im}\left[\frac{\omega_{x}+\omega_{y}-2 \omega_{n}}{\omega_{x} \omega_{y}-\omega_{n}^{2}}\right],
$$

can often be simplified. One starting point is the observation that, often, the $\omega^{(j)}$ 's are small with respect to the $\omega^{(0)}$ 's, or, as far as the real parts are concerned, are already taken into account by the $\omega^{(0)}$ 's, since they are contained in the experimental values of $\gamma_{e}, B_{a}$, and $D$. In the case of the relaxation via the real excitation of spin waves, the consequence is that the density of spin-wave states at $\omega_{n}$ is not decisively changed by the $\omega^{(j)}$ 's. The $\omega^{(j)}$ 's essentially only ensure that the imaginary parts of $\omega_{x}$ and $\omega_{y}$ do not vanish, but the exact form of those imaginary parts is not decisive. In this case, it is a good approximation to assume

$$
\begin{aligned}
& \omega_{x} \approx \omega_{x}^{(0)}-i \operatorname{sgn}\left(\omega_{n} \gamma_{e}\right) \epsilon_{x}, \\
& \omega_{y} \approx \omega_{y}^{(0)}-i \operatorname{sgn}\left(\omega_{n} \gamma_{e}\right) \epsilon_{y},
\end{aligned}
$$

where $\epsilon_{x}$ and $\epsilon_{y}$ are arbitrarily small positive numbers. Taking the limit $\epsilon_{x}, \epsilon_{y} \rightarrow 0$, one obtains

$$
F \approx 2 \pi \operatorname{sgn}\left(\omega_{n} \gamma_{e}\right) \delta\left[\left(\omega_{x}^{(0)} \omega_{y}^{(0)}\right)^{1 / 2}-\left|\omega_{n}\right|\right] c_{a}^{2},
$$

where $\delta[\cdots]$ denotes the $\delta$ function and not the skin depth, and

$$
c_{a}=\frac{1}{2}\left[\left(\frac{\omega_{x}^{(0)}}{\omega_{y}^{(0)}}\right)^{1 / 4}-\operatorname{sgn}\left(\omega_{n} \gamma_{e}\right)\left(\frac{\omega_{y}^{(0)}}{\omega_{x}^{(0)}}\right)^{1 / 4}\right] .
$$

In the case of the relaxation via the virtual excitation of spin waves, the nonvanishing spin-wave density of states at $\omega_{n}$ is due to the $\omega^{(j)}$ 's. In this case, the form of the $\operatorname{Im}\left[\omega^{(j)}\right]$ 's is decisive and must be taken into account. However, one can at least expand the real and the imaginary parts in the numerator and the denominator of $F$ into powers of $\omega^{(j)} / \omega^{(0)}$ and retain only the lowest nonvanishing order. The result is

$$
F \approx \sum_{j} \frac{-c_{x}^{2} \operatorname{Im}\left[\omega_{x}^{(j)}\right]-c_{y}^{2} \operatorname{Im}\left[\omega_{y}^{(j)}\right]}{\left(\omega_{x}^{(0)}\right)^{2}},
$$

where

$$
c_{x}=\frac{\omega_{x}^{(0)}\left(\omega_{y}^{(0)}-\omega_{n}\right)}{\omega_{x}^{(0)} \omega_{y}^{(0)}-\omega_{n}^{2}},
$$

$$
c_{y}=\frac{\omega_{x}^{(0)}\left(\omega_{x}^{(0)}-\omega_{n}\right)}{\omega_{x}^{(0)} \omega_{y}^{(0)}-\omega_{n}^{2}} .
$$

Whether the condition $\omega^{(j)} \ll \omega^{(0)}$ is fulfilled depends on the $\omega^{(j)}$ 's and on $q$. For $q \delta_{m} \leqslant 1$, it is not fulfilled, because $\omega^{(\mathrm{ed})}$ is of the order of or larger than $\omega^{(0)}$. However, for $q \delta_{m} \gg 1$, it is fulfilled, at least for the spin-wave damping mechanisms and parameters that are considered in this work, and Eqs. (26) and (28) are expected to be good approximations.

Further possibilities to simplify $F$ concern the coefficients $c_{x}$ and $c_{y}$ in Eq. (28). For many isotopes in $\mathrm{Fe}$, it is a good approximation to take the limit $\omega_{n} \ll \omega_{x}^{(0)}$, which leads to

$$
c_{x} \approx 1, \quad c_{y} \approx \frac{\omega_{x}^{(0)}}{\omega_{y}^{(0)}} .
$$

However, for the relatively high resonance frequency of ${ }^{186} \mathrm{IrFe}$ in this work, deviations of the order of several percent are expected. A further simplification can be achieved, if one takes the limit $\omega_{n} \ll \omega_{x}^{(0)} \ll \omega_{y}^{(0)}$, which leads to

$$
c_{x} \approx 1, \quad c_{y} \approx 0 .
$$

That limit applies only in the range $q l_{d} \ll 1$ and $\sin ^{2} \theta$ $\gg B_{\eta} /(4 \pi M)$, which is, however, responsible for a major part of the field dependence of the relaxation. Since it simplifies the discussion considerably, this approximation may also be applied beyond that range, but deviations from the exact result of the order of several percent are then to be expected. A further limit of interest is $\omega_{n} \ll \omega_{x}^{(0)} \approx \omega_{y}^{(0)}$, where

$$
c_{x} \approx c_{y} \approx 1,
$$

because it is a good approximation for $q l_{d} \gg 1$, that is, for the vast majority of the wave vectors.

When the formalism is applied to the spin-lattice relaxation of impurity isotopes, the question arises to which extent the modifications of the solid-state properties in the vicinity of the impurity must be taken into account. The answer follows from the involved length scales: The range of the modifications is typically of the order of a lattice constant or less. In contrast, the wave vectors that are responsible for the field dependence of $R_{\mathrm{sw}}$ are of the order of $l_{d}^{-1}$ or less, which corresponds to an effective range of the relevant interaction between the nuclear spin and the lattice of the order of $l_{d}$ or larger. The interaction thus takes place essentially in the host and is expected to be only little affected by the impurity. Accordingly, the $\omega^{(0)}$ 's and $\omega^{(j)}$ 's are approximated in this work by their values in the undisturbed host. $R_{\mathrm{sw}}$ depends on the impurity only via the hyperfine coupling constant $\left(\hbar \omega_{n}\right) / S$.

\section{B. Excitation of spin waves}

If the nuclear resonance frequency $\nu_{n}$ lies within the range of the spin-wave resonance frequencies, $R_{\mathrm{sw}}$ is essentially due to the following mechanism: The nuclear spins emit and absorb spin waves. In this work, a contribution from this relaxation mechanism can be excluded, since the lowest spin-wave frequency, $\left(\gamma_{e} B_{a}\right) /(2 \pi)=1.72 \mathrm{GHz}$, was much 
larger than $\nu_{n}$. Nevertheless, the mechanism is of interest, since under special conditions, much smaller spin-wave frequencies can occur. ${ }^{8}$

If it is assumed that the majority of the wave vectors of the emitted spin waves are much larger than $\delta_{m}^{-1}$, which should be a good approximation in many cases, Eqs. (11) and (26) can be combined to

$$
R_{\mathrm{sw}}=\frac{k_{B} 4 \pi^{2} \nu_{n}}{S} \int \frac{V c_{a}^{2}}{(2 \pi)^{3} \hbar} \delta\left[\left(\omega_{x}^{(0)} \omega_{y}^{(0)}\right)^{1 / 2}-\left|\omega_{n}\right|\right] d^{3} q,
$$

where $c_{a}$ is given by Eq. (27). Without the factor $c_{a}^{2}$, which arises from the ellipticity of the precession of the magnetization, the integral is just the density of spin-wave states at $h \nu_{n}$. Since, often, relaxation constants are derived via Fermi's golden rule instead of via the susceptibility formalism, we mention that, in this case, the ellipticity of the precession of the magnetization must already be taken into account when the spin waves are quantized. Otherwise, the factor $c_{a}^{2}$ is not reproduced.

To estimate the expected order of magnitude, $R_{\mathrm{sw}}$ was calculated according to Eq. (33) for $B_{\eta}=0$, the most favorable case, and $\nu_{n}=0.79 \mathrm{GHz}$ of ${ }^{186} \mathrm{IrFe}$. The result $R_{\mathrm{sw}}$ $=3.55 \times 10^{3}(\mathrm{~s} \mathrm{~K})^{-1}$ is about 400 times larger than $R(\infty)$ for that system. This shows that, if $\nu_{n}$ lies within the spin-wave resonance frequencies, $R_{\mathrm{sw}}$ increases the relaxation by orders of magnitude. That increase is strongly field dependent, since this is the case at all only for special choices of the direction and the magnitude of $\mathbf{B}_{\text {ext }}{ }^{8}$

\section{Excitation of sound waves}

In this section, the following relaxation mechanism is discussed: The nuclear spins virtually excite spin waves, which, in turn, decay via the excitation of sound waves. $R_{\mathrm{ph}}$ denotes the respective contribution to the relaxation constant. This mechanism can also be described in terms of the mixing of sound and spin waves in ferromagnets by the magnetostriction: ${ }^{22,29,30}$ The coupling of the nuclear spin to the spin-wave component of the sound-wave-like mode leads to spin-lattice relaxation via the excitation of the soundwave-like modes.

To derive $R_{\mathrm{ph}}$ within the susceptibility formalism, one has to solve the coupled equations of motion of the amplitude $\mathbf{m}$ of the displacement of the magnetization, which is proportional to $\exp (i \mathbf{q r}-i \omega t)$, and of the amplitude $\mathbf{u}$ of the displacement of the atoms, which is also proportional to $\exp (i \mathbf{q r}-i \omega t)$. The equation of motion of $\mathbf{u}$ is assumed to be of the form

$$
A \frac{d^{2}}{d t^{2}} \mathbf{u}=-\left(A v^{2} q^{2}\right) \mathbf{u}-\epsilon \frac{d}{d t} \mathbf{u}+\mathbf{f},
$$

where $A$ is the mass of the atom, $v$ is the speed of sound, $\epsilon$ is an arbitrarily small positive number, and $\mathbf{f}$ is the force, which is also proportional to $\exp (i \mathbf{q r}-i \omega t)$. For simplicity, it is assumed that the velocity of sound is the same for all wave vectors and polarizations.
Sound and spin waves are coupled by the magnetoelastic energy, which is, for small displacements of the magnetization from the [001] direction, of the form

$$
B_{2}\left(e_{y z} \alpha_{y}+e_{x z} \alpha_{x}\right)
$$

where $B_{2}$ is the magnetoelastic coupling constant, the $e_{i j}$ 's are the components of the strain tensor, and the $\alpha_{i}$ 's are the direction cosines of the magnetization. As a result, $\mathbf{u}$ and $\mathbf{m}$ are coupled by the following energy per atom:

$$
E_{\mathrm{me}}=-i q B_{2}\left[\left(u_{y}^{*} \cos \theta\right) \frac{m_{y}}{M}+\left(u_{x}^{*} \cos \theta+u_{z}^{*} \sin \theta\right) \frac{m_{x}}{M}\right] .
$$

It leads to the coupling of the equations of motion by the following forces and fields:

$$
\begin{gathered}
f_{j}=-\frac{\partial E_{\mathrm{me}}}{\partial u_{j}^{*}}, \\
b_{j}^{(\mathrm{ph})}=-\frac{1}{V}\left[\frac{\partial E_{\mathrm{me}}}{\partial m_{j}}\right]^{*} .
\end{gathered}
$$

With the force from Eq. (37), the solution of Eq. (34) is

$$
u_{j}=\frac{\partial E_{\mathrm{me}} / \partial u_{j}^{*}}{A\left(\omega^{2}-v^{2} q^{2}\right)+i \epsilon \omega} .
$$

Combining Eqs. (36), (38), and (39), one obtains $\mathbf{b}^{(\mathrm{ph})}$ as a function of $\mathbf{m}$. Applying, in addition, Eq. (21) and $M V$ $=\hbar\left|\gamma_{e}\right| S$, one obtains the following contribution of the sound waves to the equation of motion of the magnetization:

$$
\begin{aligned}
& \omega_{x}^{(\mathrm{ph})}=\frac{\operatorname{sgn}\left(\gamma_{e}\right) q^{2} B_{2}^{2} \cos ^{2} \theta}{\hbar S\left[A\left(\omega^{2}-v^{2} q^{2}\right)+i \epsilon \omega\right]}, \\
& \omega_{y}^{(\mathrm{ph})}=\frac{\operatorname{sgn}\left(\gamma_{e}\right) q^{2} B_{2}^{2}}{\hbar S\left[A\left(\omega^{2}-v^{2} q^{2}\right)+i \epsilon \omega\right]} .
\end{aligned}
$$

To obtain a compact expression of $R_{\mathrm{ph}}$, some additional assumptions are necessary: Neglecting all other damping mechanisms of the spin waves, all $\omega^{(j)}$ 's can be set equal to zero with the exception of the $\omega^{(\mathrm{ph})}$ 's. Moreover, assuming that the dispersion relation of the sound waves is not decisively changed by the damping and the mixing with the spin waves, one can take the limits $\epsilon \rightarrow 0$ and $B_{2}^{2} /\left(A v^{2}\right) \ll \hbar \omega_{x}^{(0)}$. Finally, assuming that $q l_{d} \ll 1$, the $q \rightarrow 0$ limit of the $\omega^{(0)}$,s can be used. If those approximations are applied together with Eq. (11), the result is

$$
R_{\mathrm{ph}}=\frac{k_{B} 2 \pi^{2} V B_{2}^{2} h \nu_{n}^{4}}{\hbar S^{2}\left(\hbar \gamma_{e} B_{\eta}\right)^{2} A v^{5}} \int_{q \rightarrow 0}\left(c_{x}^{2} \cos ^{2} \theta+c_{y}^{2}\right) \frac{d \Omega}{4 \pi},
$$

where the integration is over all directions of $\mathbf{q}$. In contrast to similar expressions in the literature, ${ }^{22,29,30} \mathrm{Eq}$. (41) takes the elliptic precession of the magnetization into account.

The decisive point is the magnitude of the effect. In $\mathrm{Fe}, B_{2}=0.57 \mathrm{meV}$ and $v$ ranges from 0.26 to 0.65 $\times 10^{6} \mathrm{~cm} \mathrm{~s}^{-1} .{ }^{23}$ For $B_{\eta}=0.059 \mathrm{~T}$ and $\nu_{n}=0.79 \mathrm{GHz}$ of ${ }^{186} \mathrm{IrFe}$, the prefactor in front of the integral in Eq. (41) 
ranges from $4.1 \times 10^{-5}$ to $4.3 \times 10^{-3}(\mathrm{~s} \mathrm{~K})^{-1}$. This is more than 3 orders of magnitude smaller than $R(\infty)$ of ${ }^{186} \mathrm{IrFe}$. Since the integral is of the order of unity, $R_{\mathrm{ph}}$ is a negligible contribution to the spin-lattice relaxation. This conclusion has already been drawn in Ref. 1, although without explicit derivation. An examination of the numerical factors shows that this result is less due to the weakness of the magnetoelastic coupling than due to the small sound-wave density of states at small $q$ 's as a result of the linear dispersion relation.

It should be added that two of the assumptions that were used to derive Eq. (41) are not good approximations. First, a uniform velocity of sound was assumed, although there is a distinct dependence of $v$ on the polarization and the propagation direction of the sound wave. The main effect of that dependence is that, actually, the factor $v^{-5}$ must be evaluated for each polarization and wave vector separately and that the different weighting of the polarizations must be taken into account. Without detailed account of the sound-wave dispersion, only an upper and a lower limit of $R_{\mathrm{ph}}$ can be obtained by inserting the minimum and the maximum $v$ into Eq. (41). Second, the neglect of the eddy-current damping is not justified. The wave vectors of the excited sound waves are in the range $q \delta_{m} \leqslant 1$, where the eddy-current damping dominates the dispersion relation of the spin waves. It can be shown that $R_{\mathrm{ph}}$ is actually considerably smaller than implied by Eq. (41), because the spin-wave amplitude is suppressed by the eddy-current damping. Thus, Eq. (41) is useful to present, in a compact expression, the decisive factors that are responsible for the negligibility of $R_{\mathrm{ph}}$, but a more elaborate expression would be needed to calculate $R_{\mathrm{ph}}$.

\section{Indirect spin-wave mechanism}

In this section, the following relaxation mechanism is discussed: The nuclear spins virtually excite spin waves, which, in turn, decay via the scattering of conduction electrons. $R_{\text {in }}$ denotes the respective contribution to the relaxation constant. This relaxation mechanism is known as the indirect spinwave mechanism, the Weger mechanism, or the second-order spin-wave mechanism. It is the dominant contribution to the spin-lattice relaxation in the rare earths. ${ }^{30}$ Its contribution to the spin-lattice relaxation in Fe has been discussed in Refs. 1,11 , and 21 . To make the relationship to the other contributions to $R_{\mathrm{sw}}$ apparent, we rederive $R_{\mathrm{in}}$ within the formalism that was developed in Sec. III A.

Since in transition metals the spin waves are excitations of the conduction electrons, it is useful to remember that at small $\omega$ 's and $q$ 's, the spin waves, as collective rotations of all spins, can be well distinguished from the scattering of the conduction electrons, which describes single electron-hole excitations. To derive $R_{\text {in }}$ within the susceptibility formalism, one has to solve the coupled equations of motion of the transverse magnetization $\mathbf{m}$ due to the spin waves and of the transverse magnetization $\mathbf{m}^{(s)}$ due to the single electron-hole excitations. The equation of motion of $\mathbf{m}^{(s)}$ is solved by

$$
\mathbf{m}^{(s)}=\frac{\chi^{(s)}}{V} \mathbf{b}^{(s)}
$$

where $\chi^{(s)}$ is the transversal susceptibility of the conduction electrons and $\mathbf{b}^{(s)}$ is the transversal field acting on $\mathbf{m}^{(s)}$. The coupling energy per atom is of the form

$$
-\frac{J V^{2}}{\gamma_{e} \gamma_{s} \hbar^{2}} \mathbf{m m}^{(s)},
$$

where $J$ is the coupling constant per unit of the involved spins and $\gamma_{s}$ is the gyromagnetic ratio of the conduction electrons. With respect to this coupling term, the interaction via the demagnetization field is negligible. The coupling gives rise to the fields

$$
\begin{gathered}
\mathbf{b}^{(s)}=\frac{J S}{\left|\gamma_{s}\right| \hbar} \frac{\mathbf{m}}{M}, \\
\mathbf{b}^{(\mathrm{in})}=\frac{J V}{\gamma_{e} \gamma_{s} \hbar^{2}} \mathbf{m}^{(s)},
\end{gathered}
$$

where $\mathbf{b}^{(\text {in) }}$ is the field acting on $\mathbf{m}$.

If Eqs. (21), (42), and (44) are combined, one obtains the following contribution of the scattering of the conduction electrons to the equation of motion of $\mathbf{m}$ :

$$
\omega_{x}^{(\mathrm{in})}=\omega_{y}^{(\mathrm{in})}=-\operatorname{sgn}\left(\gamma_{e}\right) \frac{J^{2} S}{\gamma_{s}^{2} \hbar^{3}} \chi^{(s)} .
$$

The respective contribution to the spin-lattice relaxation follows from Eqs. (11) and (28), which is expected to be a good approximation. The final result is

$$
R_{\mathrm{in}}=\frac{k_{B} \omega_{n} J^{2} V}{\hbar^{4} \gamma_{s}^{2}(2 \pi)^{3}} \int \frac{c_{x}^{2}+c_{y}^{2}}{\left(\omega_{x}^{(0)}\right)^{2}} \operatorname{Im}\left[\chi^{(s)}\right] d^{3} q .
$$

A comparison with Eq. (4) shows that $K$ is replaced in Eq. (46) by the factor $J\left(\omega_{n} / \omega_{x}^{(0)}\right)$, which can thus be interpreted as the $q$-dependent coupling constant of the indirect coupling to the conduction electrons via the magnetization. The effective range of that indirect coupling follows from the $q$ dependence of $\omega_{x}^{(0)}$ : It is of the order of $l_{d}$.

To calculate $R_{\text {in, }}$, one has to know $\operatorname{Im}\left[\chi^{(s)}\right]$, which, in turn, requires a detailed knowledge of the band structure. Since this is outside of the scope of this work, the magnitude of $R_{\text {in }}$ is left as an open problem. The estimates of $R_{\text {in }}$ in Ref. 31 are of little use, because they are unrealistic at least in the following two respects: First, those estimates are based on overestimates of the spin-lattice relaxation via the direct scattering of $s$ electrons. ${ }^{31,32}$ Second, in the case of impurity isotopes, those estimates are based on the assumption that $\operatorname{Im}\left[\chi^{(s)}\right]$ in Eq. (46) refers to the local susceptibility of the conduction electrons at the impurity. However, since the indirect coupling to the conduction electrons takes place essentially in the host, the appropriate $\operatorname{Im}\left[\chi^{(s)}\right]$ is that of the undisturbed host.

Nevertheless, some conclusions are possible without calculation. The decisive point in this work is the form of the field dependence, which can already be deduced from

$$
R_{\text {in }} \propto \int \frac{\operatorname{Im}\left[\chi^{(s)}\right]}{\left(\omega_{x}^{(0)}\right)^{2}} d^{3} q,
$$

where $c_{x}=1$ and $c_{y}=0$ was assumed for simplicity. Since $\omega_{x}^{(0)}$ is appreciably field dependent only for small $q$ 's, the knowledge of the $q$ dependence of $\operatorname{Im}\left[\chi^{(s)}\right]$ in the limit $q \rightarrow 0$ and $\omega \rightarrow 0$ is already sufficient in this context. $\operatorname{Im}\left[\chi^{(s)}\right]$ is a mea- 
sure of the resonant absorption by the scattering of conduction electrons, if a field proportional to $\exp (i \mathbf{q r}-i \omega t)$ is applied. Therefore, it is proportional to the available phase space for the scattering and to the square of the matrix element. For a given scattering mechanism and constellation of the involved bands, the resulting $q$ dependence can reasonably well be predicted. In the following, several scenarios are discussed. Since the occurrence of small momentum transfers is the prerequisite for an appreciable field dependence, only the most favorable situations in this respect are considered.

(i) One scenario, the spin-flip scattering between bands that are shifted in energy relative to each other by the exchange splitting, has already been discussed in Refs. 1, 11, and 21: In this case, there is a minimum momentum transfer $q_{m}$. $\operatorname{Im}\left[\chi^{(s)}\right]$ is proportional to $q^{-1}$ for $q>q_{m}$ and zero for $q<q_{m}$. The field dependence is of the form

$$
R_{\text {in }} \propto \frac{1}{B_{\eta}+B_{c}} .
$$

The constant $B_{c}$ is given in terms of the exchange splitting $J S$ and the gradient $\partial \epsilon / \partial q$ of the electron energy dispersion at the Fermi energy by

$$
B_{c}=\frac{D}{\hbar \gamma_{e}} \frac{(J S)^{2}}{(\partial \epsilon / \partial q)^{2}} .
$$

Without detailed knowledge of the band structure, the following can be said about $B_{c}$ : If $J S$ and $(\partial \epsilon / \partial q)$ are of the order of magnitude that can be expected for $d$ bands in Fe, $B_{c}$ is of the order of $10^{3} \mathrm{~T}$. If nearly-free-electron bands are involved, $B_{c}$ can be much smaller: Assuming a free electron energy dispersion, a Fermi energy of $8 \mathrm{eV},{ }^{33}$ and $J S$ $=0.15 \mathrm{eV},{ }^{33}$ one obtains $B_{c}=0.5 \mathrm{~T}$. However, this estimate of $B_{c}$ is already rather a lower limit.

(ii) In the case of spin-flip scattering between bands with intersecting Fermi surfaces, the available phase space approaches a nonzero value in the limit $q \rightarrow 0$. The matrix element of the spin-flip operator may or may not vanish in the limit $q \rightarrow 0$. In the latter case, $\operatorname{Im}\left[\chi^{(s)}\right]$ is approximately constant for small $q$ 's. Applying Eq. (47), it can be shown that in this case, the field dependence is of the form $R_{\text {in }} \propto B_{\eta}^{-1 / 2}$.

(iii) Scattering that involves a change of the orbital magnetic quantum number instead of a spin-flip can also contribute to $R_{\text {in }}$. However, the required coupling between the magnetization and the orbital moment cannot be the spin-orbit coupling, since it must be an electron-electron interaction. Instead, it may arise from the intra-atomic interaction between the orbital moment that is admixed to the magnetization by the spin-orbit coupling and the orbital moment of the scattered electron. The most favorable constellation is the scattering into the same band. In this case, the available phase space is proportional to $q^{-1}$, whereas the square of the matrix element of the orbital moment raising operator is proportional to $q^{2}$. As a result, $\operatorname{Im}\left[\chi^{(s)}\right] \propto q$. Applying Eq. (47), it can be shown that in this case,

$$
R_{\text {in }} \propto \log \left[\frac{B_{c}}{B_{\eta}}\right],
$$

where $B_{c}$ is at least of the order of $50 \mathrm{~T}$.

For comparison, according to our measurements, the field-dependent part of the spin-lattice relaxation can be well described by a term that is proportional to $B_{\eta}^{-\xi}$, where $\xi$ is close to 1.4, if $B_{\eta}$ is of the order of $0.1 \mathrm{~T}$. This observed field dependence is much stronger than any of the predicted field dependences of $R_{\text {in }}$. Therefore, the conclusion is that the indirect spin-wave mechanism cannot explain the magneticfield dependence of the spin-lattice relaxation, at least not with the scattering mechanisms and band structure constellations that are known to us.

Finally, it should be mentioned that, for simplicity, our derivation of the field dependence of the indirect spin-wave mechanism neglects the following two effects, which may somewhat modify the form of the field dependence: The ellipticity of the precession of the magnetization is neglected by the assumptions $c_{x}=1$ and $c_{y}=0$. Moreover, in addition to the contributions from the direct and from the indirect coupling to the conduction electrons, the superposition of both couplings also contributes to the spin-lattice relaxation. This contribution, which is discussed in Refs. 1 and 11, is neglected in this work. However, it can be shown that both effects, at most, lead to an even weaker field dependence. Our conclusion that the field dependence of the indirect spinwave mechanism is too weak is thus not affected.

\section{E. Eddy-current damping}

In this section, the following relaxation mechanism is discussed: The nuclear spins virtually excite spin waves, which, in turn, induce eddy currents, which, in turn, decay via the processes that are summarized by the term electrical resistivity. $R_{\text {ed }}$ denotes the respective contribution to the relaxation constant. The contribution of the eddy currents to the equation of motion of the magnetization has already been derived in Sec. III A, where it is specified in terms of the $\omega^{(\mathrm{ed})}$ 's by

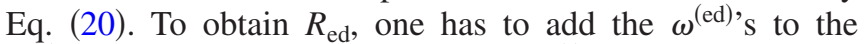
$\omega^{(0)}$ 's, whereas the influence of other $\omega^{(j)}$ 's can be neglected, because for $q \delta_{m} \gg 1$, all contributions to the relaxation add independently, and for smaller $q$ 's, the other $\omega^{(j)}$ 's are negligible with respect to the $\omega^{\text {(ed)'s. }}$

It follows that $R_{\text {ed }}$ is given by Eq. (11) with

$$
\begin{gathered}
\omega_{x}=\gamma_{e}\left(B_{\eta}+\frac{D q^{2}}{\left|\gamma_{e}\right| \hbar}+4 \pi M \frac{2}{2+i \operatorname{sgn}(\omega) \delta^{2} q^{2}}\right), \\
\omega_{y}=\gamma_{e}\left(B_{\eta}+\frac{D q^{2}}{\left|\gamma_{e}\right| \hbar}+4 \pi M \frac{2+i \operatorname{sgn}(\omega)(\sin \theta)^{2} \delta^{2} q^{2}}{2+i \operatorname{sgn}(\omega) \delta^{2} q^{2}}\right),
\end{gathered}
$$

where $\delta$ is given as a function of $\mathbf{q}$ and $\omega=\omega_{n}$ by the Eqs. (16) and (22).

\section{Approximate expression}

To reproduce $R_{\text {ed }}$ within $1 \%$, the numerical evaluation of the set of Eqs. (11), (16), (22), and (51) is unavoidable. How- 
ever, a less involved, though only approximate expression was also derived. To understand the used approximations, it is useful to discuss first the $q$ dependence of the integrand in Eq. (11). In the decisive range of $q$ 's, that integrand is largely given by $\operatorname{Im}\left(\omega_{x}^{-1}\right)$, which is proportional to $(q \delta)^{2}$ for $q \delta_{m} \ll 1$, passes through a maximum at $q \delta_{m}=1$, and is proportional to $(q \delta)^{-2}\left[1+\left(q l_{d}\right)^{2}\right]^{-2}$ for $q \delta_{m} \gg 1$. It follows that $R_{\text {ed }}$ is mainly due to momentum transfers in the range $\delta_{m}^{-1}<q<l_{d}^{-1}$.

The following three approximations were applied: First, the integrand was approximated by Eq. (28), the appropriate expression for $q \delta_{m} \gg 1$, and was integrated from $q \delta_{m}=1$ to $q=\infty$. Second, $c_{x}=1$ and $c_{y}=0$ was assumed, which corresponds to taking the limits $\omega_{n} \ll \omega_{x}^{(0)}$ and $\omega_{x}^{(0)} \ll \omega_{y}^{(0)}$. Third, $\sigma$ was approximated by its expression in the limit $q \Lambda \gg 1$ :

$$
\sigma \approx \sigma_{0} \frac{3 \pi}{4 \Lambda q} .
$$

This is justified for large resistivity ratios, the criterion being $\Lambda>\delta_{m}$.

If all three approximations are combined, $R_{\text {ed }}$ can be expressed in closed form. For convenience, we give the final result in terms of the numerical values of the involved quantities:

$$
\begin{aligned}
R_{\mathrm{ed}} \approx & 6.18 \times 10^{-3} \frac{V 4 \pi M}{\left(\left|\gamma_{e}\right| / 2 \pi\right) S} \frac{\nu_{n}^{2}}{B_{\eta}^{2}} \frac{\sigma_{0}}{\Lambda} \\
& \times\left\{\log \left[2.73 \times 10^{5} \frac{\left(\left|\gamma_{e}\right| / 2 \pi\right)}{D(4 \pi M)^{2 / 3}} \frac{B_{\eta}^{5 / 3}}{\nu_{n}^{2 / 3}}\left(\frac{\sigma_{0}}{\Lambda}\right)^{-2 / 3}\right]-1\right\} .
\end{aligned}
$$

This expression applies if $R$ is expressed in $(\mathrm{s} \mathrm{K})^{-1}, V$ in $\AA^{3}$, $4 \pi M$ and $B_{\eta}$ in T, $\nu_{n}$ in $\mathrm{GHz},\left(\gamma_{e} / 2 \pi\right)$ in $\mathrm{GHz} \mathrm{T}^{-1}, D$ in $\mathrm{meV} \AA^{2}$, and $\left(\sigma_{0} / \Lambda\right)$ in $(\mu \Omega \mathrm{cm} \mu \mathrm{m})^{-1}$. To examine the typical agreement of Eq. (53) with the exact expression, the results were compared for the parameter sets that were used in this work to describe the relaxation of ${ }^{186} \mathrm{Ir}$. The deviation was in all cases less than $10 \%$.

\section{Properties}

In the following, the properties of the spin-lattice relaxation via the eddy-current damping are discussed. The first property is the magnitude of the effect. It can be inferred using Eq. (53). The main uncertainty arises from $\sigma_{0} / \Lambda$. That parameter, which is independent of the mean free path, is essentially the product of the mobility and the density of the conduction electrons. It is an intrinsic property of the host. Still, the best way to obtain a realistic estimate for $\mathrm{Fe}$ is to consider the values for other metals: The order of magnitude of $\sigma_{0} / \Lambda$ should be the same for transition metals as for other metals, because the larger densities of electrons at the Fermi energy are expected to be compensated by correspondingly smaller mobilities. For $\mathrm{Al}, \mathrm{Sn}, \mathrm{Cd}, \mathrm{Pb}, \mathrm{Cu}, \mathrm{Ag}$, and $\mathrm{Au}$, values of $\sigma_{0} / \Lambda$ between 5.7 and $20.4(\mu \Omega \mathrm{cm} \mu \mathrm{m})^{-1}$ have been reported. ${ }^{34}$ Assuming that $\sigma_{0} / \Lambda$ of $\mathrm{Fe}$ lies within that range, $R_{\text {ed }}=16-41(\mathrm{~s} \mathrm{~K})^{-1}$ is obtained from Eq. (53) for $\nu_{n}$ $=0.79 \mathrm{GHz}$ and $B_{\eta}=0.059 \mathrm{~T}$, which applies to ${ }^{186} \mathrm{Ir}$ in Fe at zero applied field. This has to be compared with the observed magnitude of the field-dependent part of the relaxation in that case, $R(0)-R(\infty)=24(\mathrm{~s} \mathrm{~K})^{-1}$. $R_{\text {ed }}$ is thus of the right order of magnitude to explain the field dependence of the relaxation.

The second property that we discuss is the independence from the impurity. $R_{\text {ed }}$ depends only on properties of the host and $\nu_{n}$, but not on the element to which the particular isotope belongs or on the lattice site that it occupies. That independence from the local electronic structure reflects the long effective range of the interaction with the lattice, which also manifests itself in the dominance of small momentum transfers. However, it is not a distinctive feature of $R_{\text {ed }}$, since every close relation between the relaxation and $B_{\eta}$, a quantity that describes the response of the system to macroscopic perturbations, suggests a long-range interaction with the electrons.

The third property that we discuss is the form of the magnetic-field dependence. That dependence is actually a $B_{\eta}$ dependence, since $B_{\text {ext }}$ enters only via that quantity. There is a proportionality to $B_{\eta}^{-2}$, which is, however, weakened by the field dependences of $\delta_{m}$ and $l_{d}$. That weakening, represented by the $B_{\eta}$ dependence of the log term in Eq. (53), increases with decreasing magnetic field but also depends on the other parameters. For example, for the parameters that were used in this work, $R_{\text {ed }}$ is proportional to $B_{\eta}^{-1.47}$ at $B_{\eta}=0.059 \mathrm{~T}$, to $B_{\eta}^{-1.59}$ at $B_{\eta}=0.12 \mathrm{~T}$, and to $B_{\eta}^{-1.75}$ at $B_{\eta}=1.0 \mathrm{~T}$, if the field dependence is described over small field ranges as a power law in $B_{\eta}$.

The fourth property that we discuss is the dependence on the nuclear resonance frequency. $R_{\text {ed }}$ is roughly proportional to $\nu_{n}^{2}$. This corresponds to the usual scaling of the nuclear spin-lattice relaxation with the square of the relevant hyperfine coupling constant, which is, in our case, the static hyperfine interaction.

However, there are also slight, but distinct deviations from $R_{\text {ed }} \propto \nu_{n}^{2}$. Three effects can be distinguished in this respect: First, the skin effect, which suppresses displacements of the magnetization with wavelengths larger than $\delta$, is less effective at smaller frequencies. Due to that effect, which gives rise to the $\nu_{n}$ dependence of the logarithmic term in Eq. (53), $R_{\mathrm{ed}} / \nu_{n}^{2}$ increases with decreasing $\nu_{n}$. Second, the integrand of Eq. (11) becomes almost singular at $\omega_{n}^{2}=\omega_{x}^{(0)} \omega_{y}^{(0)}$. Therefore, $R_{\text {ed }} / \nu_{n}^{2}$ increases when $\left|\omega_{n}\right|$ approaches the range of spin-wave precession frequencies at $\left(\omega_{x}^{(0)} \omega_{y}^{(0)}\right)^{1 / 2}$. Third, there is an asymmetry with respect to the sign of the frequency: The relaxation is faster if the free precessions of the nuclear and the electron spins have the same sense. The relative sense of those precessions was specified in terms of the signs of $\gamma_{e}, \gamma_{n}$, and $B_{\mathrm{HF}}$ in connection with Eq. (6). The combined effect of all three effects is illustrated in Fig. 1.

The fifth property that we discuss is the dependence on the conductivity, which is the product of the host-specific parameter $\sigma_{0} / \Lambda$ and the mean free path $\Lambda$, which varies with 


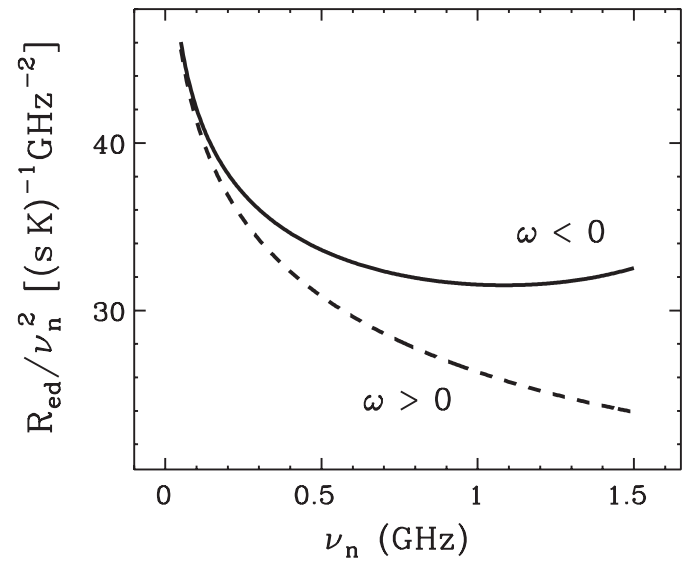

FIG. 1. Dependence of $R_{\text {ed }} / \nu_{n}^{2}$ on $\nu_{n}$ for $B_{\eta}=0.059 \mathrm{~T}, \sigma_{0} / \Lambda$ $=6.7(\mu \Omega \mathrm{cm} \mu \mathrm{m})^{-1}, \sigma_{0}=20(\mu \Omega \mathrm{cm})^{-1}$, and Fe as the host. $\omega>0$ denotes that the electronic and the nuclear spins precess in the opposite sense, and $\omega<0$ that they precess in the same sense.

the temperature and the sample preparation. As can be seen from Eq. (53), the dependence of $R_{\text {ed }}$ on $\sigma_{0} / \Lambda$ is essentially given by a proportionality to $\sigma_{0} / \Lambda$, which is only slightly weakened by the $\sigma_{0} / \Lambda$ dependence of the logarithmic term. This reflects that the relaxation is proportional to the eddycurrent damping, apart from small momentum transfers $\left(q<\delta_{m}^{-1}\right)$, where the damping becomes so large that it suppresses the susceptibility.

The dependence on $\Lambda$ is more complex, since the conductivity becomes independent of $\Lambda$, if $\Lambda$ becomes larger than the wavelength. Therefore, $\Lambda$ must be compared with the relevant length scales of the problem, $l_{d}$ and $\delta_{m}$. As long as $\Lambda \ll l_{d}, R_{\text {ed }}$ is largely proportional to $\Lambda$. In the range $l_{d}<\Lambda$ $<\delta_{m}$, the increase with $\Lambda$ becomes ever weaker, and in the opposite limit, $\Lambda \gg \delta_{m}, R_{\text {ed }}$ is independent of $\Lambda$.

The resulting dependence of $R_{\text {ed }}$ on $\Lambda$ is shown in Fig. 2. There, the resistivity ratio $\sigma_{0} / \sigma_{0}(300 \mathrm{~K})$ serves as the measure of $\Lambda$. It can be seen that $R_{\text {ed }}$ is rather insensitive to $\Lambda$, as long as $\sigma_{0} / \sigma_{0}(300 \mathrm{~K})>30$, which is fulfilled for well prepared samples at low temperatures.

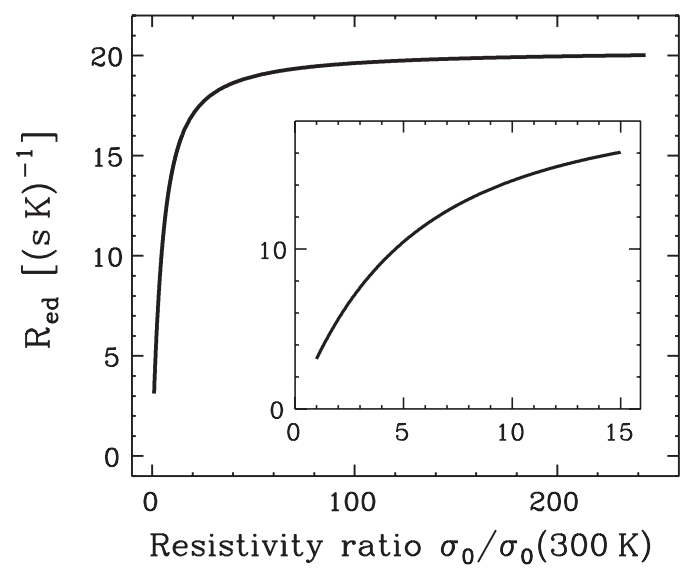

FIG. 2. Dependence of $R_{\text {ed }}$ on the resistivity ratio for $B_{\eta}$ $=0.059 \mathrm{~T}, \quad \nu_{n}=0.79 \mathrm{GHz}, \quad \sigma_{0} / \Lambda=6.7(\mu \Omega \mathrm{cm} \mu \mathrm{m})^{-1}, \quad \sigma_{0}(300 \mathrm{~K})$ $=0.1(\mu \Omega \mathrm{cm})^{-1}$, and $\mathrm{Fe}$ as the host. The region of small resistivity ratios is shown enlarged in the inset.
The sixth property that we discuss is the temperature dependence. The usual proportionality of the spin-lattice relaxation in metals to $T$ is already taken into account by the definition of $R_{\text {ed }}$. Additional temperature dependences arise from the temperature dependences of $\Lambda, B_{a}$, and $D$. Since the last two parameters vary only weakly up to room temperature, the temperature dependence of $R_{\text {ed }}$ is essentially determined by the temperature dependence of $\Lambda$. Accordingly, the curve in Fig. 2 can also be viewed as a plot of the temperature dependence if $\sigma_{0}(T) / \sigma_{0}(300 \mathrm{~K})$ is interpreted as a measure of $T$.

The basic effect is that $\Lambda$ and $R_{\text {ed }}$ decrease with increasing temperature. However, due to the insensitivity of $R_{\text {ed }}$ to $\Lambda$ at high resistivity ratios, the decrease of $R_{\text {ed }}$ sets in later than the decrease of $\Lambda$. Taking the example of Fig. 2, the onset of an appreciable temperature dependence of $R_{\text {ed }}$ is expected at $\sigma_{0}(T) / \sigma_{0}(300 \mathrm{~K}) \sim 30$, which corresponds to $T \sim 65 \mathrm{~K} .{ }^{35} \mathrm{At}$ room temperature, $R_{\text {ed }}$ is already reduced by more than a factor of 5 .

The last property that we discuss is the influence of the surface. Surface effects come into play when the distance to the surface becomes smaller than the skin depth, which is necessarily the case with NMR measurements. They arise because additional magnetic surface anisotropy terms and the missing magnetic volume anisotropy at the other side of the surface modify the susceptibility, and because the truncation of the free path of the conduction electrons at the surface modifies the conductivity. We do not give a detailed treatment because the required mathematical techniques, such as the Wiener-Hopf technique, ${ }^{27}$ are beyond our scope. Moreover, decisive parameters, such as the magnetic surface anisotropy, are, in general, not known.

However, several general conclusions can already be drawn assuming strongly simplified boundary conditions. If surface effects on the conductivity are completely ignored, whereas the magnetic surface anisotropy is assumed to be either absent (free-spin boundary condition) or so strong that the magnetization at the surface cannot be displaced at all (pinned-spin boundary condition), the problem can be solved by the introduction of a mirror nuclear spin. The result is that the integrand in Eq. (11) must be multiplied by an extra factor

$$
1 \pm \cos (2 \mathbf{q d})
$$

where $\mathbf{d}$ is the distance to the surface, and the plus and the minus signs apply to the free-spin and the pinned-spin limits, respectively. This shows that the surface contribution to $R_{\text {ed }}$ (i) can become of the same order of magnitude as the volume contribution, (ii) can enhance or reduce the relaxation, and (iii) depends on the surface conditions and thus on the sample preparation.

With regard to the range of the surface effects into the interior of the sample, the following can be said without detailed theory: The characteristic length scales of the interaction between the nuclear spin and the lattice are $\delta_{m}$ and $l_{d}$. Accordingly, the surface effects are largest for $d<l_{d}$, diminish with increasing distance to the surface in the range $l_{d}<d<\delta_{m}$, and can be neglected for $d>\delta_{m}$. 


\section{F. Gilbert damping}

In this section, the following relaxation mechanism is discussed: The nuclear spins virtually excite spin waves, which, in turn, decay via the Gilbert damping. $R_{\mathrm{gi}}$ denotes the respective contribution to the relaxation constant. Since the mechanisms of the Gilbert damping are not known, in this way, the relaxation mechanism is specified only in part, in part it is described only phenomenologically. For example, it may well be that the scattering of conduction electrons by the spin waves is part of the Gilbert damping and that $R_{\text {in }}$ is part of $R_{\mathrm{gi}}$. The advantage of the phenomenological concept of the Gilbert damping is that it is the generally accepted description of the damping of the precession of the magnetization, which has been applied, for example, to many ferromagnetic-resonance experiments.

The contribution of the Gilbert damping to $d \mathbf{M} / d t$ is

$$
-\frac{G}{\gamma_{e} M^{2}}\left(\mathbf{M} \times \frac{d \mathbf{M}}{d t}\right),
$$

where $G$ is the Gilbert damping parameter. Here, $G$ is assumed to be independent of $q$. This is in accord with the use of $G$ in the literature, where it is treated as a constant, irrespective of the length scales of the problem, such as the skin depth or the thickness of thin films. However, one should be aware that the $q$ independence of $G$ is not well established and that a $q$ dependence would distinctly alter the properties of $R_{\mathrm{gi}}$.

In passing, we note that the form of the Gilbert damping might appear somewhat peculiar, if compared, for example, to a Bloch-type damping: (i) The relaxation of a displacement of the magnetization is proportional to the velocity and not to the magnitude of the displacement. (ii) In the case of an elliptic precession of the magnetization, the relaxation is, in general, not directed toward the equilibrium position. (iii) If the magnetization precesses in a sense that is opposite to the sense of the free precession, which may occur in response to an external rf field, the damping term increases the displacement of the magnetization. In this context, it is of interest that it can be shown that the eddy-current damping also shows all those peculiarities.

It can be shown that the contributions to $\omega_{x}$ and $\omega_{y}$ from the Gilbert damping are

$$
\omega_{x}^{(\mathrm{gi})}=\omega_{y}^{(\mathrm{gi})}=-i \frac{G \omega}{\gamma_{e} M} .
$$

Since it turns out that $R_{\mathrm{gi}}$ is mainly due to momentum transfers of the order of $q \sim l_{d}^{-1}$, approximation (28) can be applied. The result is

$$
R_{\mathrm{gi}}=\frac{k_{B} \omega_{n}^{2} V}{\hbar S(2 \pi)^{3}} \frac{G}{\left|\gamma_{e}\right| M} \int \frac{c_{x}^{2}+c_{y}^{2}}{\left(\omega_{x}^{(0)}\right)^{2}} d^{3} q
$$

If $\omega_{n} \ll \omega_{x}^{(0)}$, Eq. (57) can be further simplified. In that limit, the integrand reduces to

$$
\left(\omega_{x}^{(0)}\right)^{-2}+\left(\omega_{y}^{(0)}\right)^{-2}
$$

which can be integrated by standard integrals. The result is

$$
R_{\mathrm{gi}} \approx \frac{k_{B} \hbar^{1 / 2} V \omega_{n}^{2} G}{8 \pi S\left(D \gamma_{e}\right)^{3 / 2} M B_{\eta}^{1 / 2}} F_{c}\left(\frac{B_{\eta}}{4 \pi M}\right),
$$

where

$$
F_{c}(x)=1+x^{1 / 2} \arcsin \left(\frac{1}{(1+x)^{1 / 2}}\right) .
$$

The characteristic length and wavelength scales of the interaction between the nuclear spin and the medium are reflected by the $q$ dependence of the integrand in Eq. (57), which is largely proportional to $\left[1+\left(q l_{d}\right)^{2}\right]^{-2}$. It follows that the length scale is essentially given by $l_{d}$ and that mainly momentum transfers of the order of $q \sim l_{d}^{-1}$ are involved. In comparison to $R_{\text {ed }}$, where the relevant length scales are $\delta_{m}$ and $l_{d}$, very small momentum transfers and very large distances are less involved.

The magnitude of $R_{\mathrm{gi}}$ can be calculated taking $G$ $=0.053-0.076 \mathrm{GHz}$ from the literature. ${ }^{36}$ For $\nu_{n}=0.79 \mathrm{GHz}$ and $B_{\eta}=0.059 \mathrm{~T}$, which applies to ${ }^{186} \mathrm{Ir}$ in Fe at zero applied field, one obtains $R_{\mathrm{gi}}=3.2-4.6(\mathrm{~s} \mathrm{~K})^{-1}$. The comparison with the experimental relaxation constants, $R(0)-R(\infty)$ $=24(\mathrm{~s} \mathrm{~K})^{-1}$ and $R(\infty)=8(\mathrm{~s} \mathrm{~K})^{-1}$, shows that $R_{\mathrm{gi}}$ is a nonnegligible contribution to the field-dependent part of the relaxation, although it is not the main contribution. The element- and lattice-site-specific local electronic structure at the impurity does not enter except via the parameter $\omega_{n}$. That impurity independence results, as in the case of $R_{\text {ed }}$, from the long range of the interaction between the spin and the medium.

The magnetic-field dependence of $R_{\mathrm{gi}}$ is determined by the factors $B_{\eta}^{-1 / 2}$ and $F_{c}\left[B_{\eta} /(4 \pi M)\right]$ in Eq. (59). The last factor distinctly weakens the proportionality to $B_{\eta}^{-1 / 2}$ at moderate field strengths. Between $B_{\eta}=0.059 \mathrm{~T}$ and $B_{\eta}=2 \mathrm{~T}$, for example, the field dependence of $R_{\mathrm{gi}}$ can be well described by $B_{\eta}^{-\xi}$ with $\xi$ close to 0.40 .

The dependence of $R_{\mathrm{gi}}$ on the nuclear resonance frequency largely follows $R_{\mathrm{gi}} \propto \nu_{n}^{2}$. The deviations from that proportionality are distinctly smaller than in the case of $R_{\text {ed }}$ : At $B_{\eta}=0.059 \mathrm{~T}$, for example, $R_{\mathrm{gi}} / \nu_{n}^{2}$ increases by $5.9 \%$ between $\nu_{n}=0 \mathrm{GHz}$ and $\nu_{n}=1 \mathrm{GHz}$ if the electronic and the nuclear spins precess in the same sense and decreases by $3.6 \%$ if the electronic and the nuclear spins precess in the opposite sense.

The temperature dependence of $R_{\mathrm{gi}}$ is only weak up to room temperature, since the parameters $G, B_{a}, D, M, \gamma_{e}$, and $V$ are only weakly temperature dependent. Surface effects are introduced by the magnetic surface anisotropy and the missing magnetic volume anisotropy beyond the surface. They may become as important as in the case of $R_{\text {ed }}$. However, the distance to the surface where they become important is of the order of $l_{d}$ and thus much smaller than in the case of $R_{\text {ed }}$, where it is of the order of $\delta_{m}$.

\section{G. Domain walls}

The nuclear spin-lattice relaxation in the domain walls is known to be by up to 2 orders of magnitude faster than the relaxation in the domains. ${ }^{21,37,38}$ This had no consequences 
for the experiments in this work, since the used measurement technique is insensitive to the nuclei in the domain walls. Nevertheless, a short comment is appropriate, since this is a case where the ferromagnetism causes a strong enhancement of the relaxation and where the origin of this enhancement is thought to be known.

It has been proposed that the nuclear spins in the domain walls couple to vibrations of the domain walls, which, in turn, are damped by eddy currents. ${ }^{21,39}$ Thus, it might be possible to develop a unified treatment of the relaxation in domain walls and the relaxation via eddy currents. One difficulty will be that in the case of the domain walls, their spatial distribution and their restoring forces play an important role. The poor knowledge of those parameters will make a detailed comparison with the experiment difficult.

\section{RELAXATION MEASUREMENT BY NUCLEAR MAGNETIC RESONANCE ON ORIENTED NUCLEI}

In nuclear magnetic resonance on oriented nuclei (NMR$\mathrm{ON}$ ), the resonant depolarization of the radioactive probe nuclei is detected via the resulting change in the anisotropic emission of the $\gamma$ radiation. ${ }^{40}$ To measure the nuclear spinlattice relaxation by $\mathrm{NMR}-\mathrm{ON}$, the frequency modulation (FM) of the rf field is periodically switched on and off. 8,41 Due to the inhomogeneous broadening of the resonance, the nuclear spins are excited only if the FM is enabled and relax back to thermal equilibrium if it is switched off.

Essentially, three parameters can be obtained from a least squares fit to the relaxation curve of the $\gamma$ anisotropy during the FM on-off cycle: the relaxation constant $R$, the rf transition rate $R_{\mathrm{rf}}$, which is defined below, and the fraction $f_{\mathrm{rf}}$ of the probe nuclei that are excited by the FM. $f_{\text {rf }}<1$ occurs, for example, if some probe nuclei are located on slightly disturbed lattice sites, with resonance frequencies that lie outside of the bandwidth of the FM.

At the low temperatures of NMR-ON experiments, a multiexponential relaxation behavior is observed, which can be described by a set of rate equations (the master equation) for the sublevel populations:

$$
\frac{d}{d t} p_{m}=\sum_{n}\left(W_{m, n} p_{n}-W_{n, m} p_{m}\right),
$$

where $p_{m}$ is the population of the level with the magnetic quantum number $m$ and $W_{m, n}$ is the transition rate from the level $n$ to the level $m$. The transition rates are given by

$$
\begin{aligned}
& W_{m+1, m}=c_{m, m+1}\left[\frac{h \nu_{n}}{2 k_{B}(1-b)} R+R_{\mathrm{rf}}\right], \\
& W_{m, m+1}=c_{m, m+1}\left[\frac{h \nu_{n} b}{2 k_{B}(1-b)} R+R_{\mathrm{rf}}\right],
\end{aligned}
$$

where

$$
c_{m, m+1}=I(I+1)-m(m+1),
$$

$$
b=\exp \left(\frac{-h \nu_{n}}{k_{B} T}\right),
$$

and $I$ is the nuclear spin. When the FM is not applied, $R_{\mathrm{rf}}=0$. We only mention that actually more sophisticated expressions for the transition rates were used, which are given in Ref. 42. However, the differences are not decisive in the context of this work. The solution of the master equation and the relationship between the sublevel populations and the $\gamma$ anisotropy are described in detail in Refs. 3, 41, and 43.

The description of the relaxation behavior of dilute nuclear spins by transition rates is well established in the absence of a rf field. The use of the $\mathrm{rf}$ transition rate $R_{\mathrm{rf}}$, however, has been discussed controversially: On one hand, it has been argued that the effect of a coherent rf field cannot correctly be treated in that way. ${ }^{2,3,12,14}$ On the other hand, it has been argued that in NMR-ON experiments, the coherence is sufficiently disturbed to justify such a treatment. $9,44,45$ To clarify that point, in the remainder of this section, the excitation process is analyzed in more detail.

Due to the FM, the rf field induces transitions between the sublevels only during small time intervals when the rf frequency passes the resonance frequency of the particular spin. Fast passages, therefore, alternate with intervals of nearly free precession. Within the rotating frame, each passage causes a rotation of the spins by an angle $\theta$ around the $y$ axis, whereas in the time until the next passage, the spins precess around the $z$ axis by the angle $\phi$. $\theta$ depends on the rf-field strength. Usually, $\theta \ll \pi$. In that limit, $\theta^{2}$ is proportional to the applied rf power per FM bandwidth. $\phi$ is given by

$$
\phi=2 \pi \int_{t_{p}}^{t_{p}+\Delta t}\left[\nu_{n}-\nu_{\mathrm{rf}}(t)\right] d t,
$$

where $\nu_{\mathrm{rf}}(t)$ is the frequency of the rf field as a function of the time, $t_{p}$ is the time of the passage, and $\Delta t$ is the time between successive passages. Contributions of the fast passages to $\phi$ are neglected here for simplicity, because they change the dependences of $\phi$ on $\nu_{n}$ and $\nu_{\text {rf }}$ not decisively.

To describe the sequence of the rotations of the spins, it is convenient to expand the spin density matrix $\rho$ into irreducible tensor operators $T_{l m}$ of rank $l$ and order $m$ according to

$$
\rho=\sum_{l=0}^{2 I} \sum_{m=-l}^{l} b_{l m} T_{l m},
$$

where the $b_{l m}$ 's are complex coefficients. ${ }^{46,47}$ The coefficients $b_{l m}^{(j+1)}$ before the $(j+1)$ th passage are then given in terms of the coefficients before the $j$ th passage by ${ }^{48}$

$$
b_{l m}^{(j+1)}=\exp (-i m \phi) \sum_{m^{\prime}=-l}^{l} d_{m m^{\prime}}^{(l)}(\theta) b_{l m^{\prime}}^{(j)} .
$$

Here, the $d_{m m^{\prime}}^{(l)}(\theta)$ 's are the elements of the reduced rotation matrix, which are given, for example, in Ref. 48.

$\phi$ is the sum of its nominal value $\phi_{0}$ and of a fluctuating part $\phi_{f}$, which varies from passage to passage, because the instability of the rf generator leads to small fluctuations of $\nu_{\mathrm{rf}}$ around its nominal value. For example, for a sawtooth modulation, Eq. (63) gives 


$$
\phi_{0}=2 \pi \frac{\nu_{n}-\nu_{c}}{\nu_{\mathrm{FM}}},
$$

where $\nu_{\mathrm{FM}}$ is the modulation frequency and $\nu_{c}$ the center frequency. Since the inhomogeneous broadening of $\nu_{n}$ is usually much larger than $\nu_{\mathrm{FM}}$, all values of $\phi_{0}$ between 0 and $2 \pi$ occur almost equally frequently. The mean square deviation $\Delta \phi_{f}$ of $\phi_{f}$ can be related to the full width of half maximum $\Delta \nu_{\mathrm{rf}}$ of the frequency spectrum of the fluctuations of $\nu_{\mathrm{rf}}$. If the correlation time of the fluctuations is much smaller than both $\nu_{\mathrm{FM}}^{-1}$ and $\left(\Delta \nu_{\mathrm{rf}}\right)^{-1}$, the spectrum is motion narrowed, ${ }^{49}$ and the following relation can be derived from Eq. (63):

$$
\Delta \phi_{f}=\left(2 \pi \frac{\Delta \nu_{\mathrm{rf}}}{\nu_{\mathrm{FM}}}\right)^{1 / 2} .
$$

This detailed description of the excitation process can be used to calculate the temporal evolution of the density matrix after the FM is switched on. Initially, the density matrix is diagonal, which implies that only the $b_{l 0}$ 's are different from zero. The subsequent changes of the $b_{l m}$ 's from passage to passage follow from Eq. (65). To take the variation of $\phi_{0}$ between 0 and $2 \pi$ into account, one must either repeat the calculation for different $\phi_{0}$ 's and take the average as the density matrix of the entire spin system. Or one expands the $b_{l m}$ 's into powers of $\exp \left(i \phi_{0}\right)$, calculates the temporal evolution of the expansion coefficients by a correspondingly extended version of Eq. (65), and takes the coefficients of the zeroth power as the density matrix of the entire spin system.

To simulate the fluctuations of $\phi_{f}$, before each passage, a new value of $\phi_{f}$ was determined by a random number generator in such a way that the probability distribution of $\phi_{f}$ was Gaussian with mean square deviation $\Delta \phi_{f}$. For simplicity, it was assumed that $\phi_{f}$ is the same for all spins. Due to the fluctuations of $\phi_{f}$, the excitation curves fluctuate too. However, in the measurements, these fluctuations are reduced, because the average over several FM on-off cycles is taken. Accordingly, the evolution of $\rho$ was calculated a number of $n_{r}$ times with different random numbers, and the average was adopted as the final result.

In this way, the exact temporal evolution of the spin density matrix was calculated for different values of $l, \theta, \Delta \phi_{f}$, and $n_{r}$. The comparison with the predictions of the rate equations (61) and (62) showed under which conditions those equations are a good approximation. If the spin-lattice relaxation is neglected, the rate equations, which describe only the diagonal part of $\rho,{ }^{47}$ predict $^{41}$

$$
\begin{gathered}
b_{l 0}(t)=b_{l 0}(0) \exp \left(-k_{l} t\right), \\
k_{l}=l(l+1) R_{\mathrm{rf}} .
\end{gathered}
$$

This turned out to be a good approximation under the following conditions.

(i) The fluctuations of the $b_{l 0}^{(j)}$ 's due to the fluctuations of $\phi_{f}$ are roughly proportional to $\left(n_{l} n_{r}\right)^{-1 / 2}$, where $n_{l}$ is the number of the fast passages after which $b_{l 0}$ is reduced by a factor of $e . n_{l} n_{r}$ can be interpreted as the number of $\phi_{f}$ 's that contribute to the essential part of the excitation curve. In order that the temporal evolution of $b_{l 0}$ is reasonably smooth and well defined, that number must be large enough. (ii) The coherence between the rf field and the spin system is disturbed by the random variations of $\phi_{0}$ from spin to spin and of $\phi_{f}$ from passage to passage. In order that the coherence gets essentially lost, $\Delta \phi_{f}$ must be at least of the order of $\pi$. In that case, the individual contributions to the nondiagonal elements of $\rho$ cancel each other, and the diagonal elements decay, in the limit $n_{r} \rightarrow \infty$, exponentially according to

$$
b_{l 0}^{(j+1)}=b_{l 0}^{(1)}\left[d_{00}^{(l)}(\theta)\right]^{j} .
$$

(iii) In order that the respective decay constants are proportional to $l(l+1)$ and to the applied rf power, the condition $\theta \ll \pi$, which is equivalent to $n_{l} \gg 1$, must be fulfilled. In that limit the decay constant of $b_{l 0}$ can be approximated by

$$
\frac{-\log \left[d_{00}^{(l)}(\theta)\right]}{\Delta t} \approx \frac{l(l+1) \theta^{2}}{4 \Delta t},
$$

which is identical to $k_{l}$ of Eq. (68), if $R_{\mathrm{rf}}$ is identified with $\theta^{2} /(4 \Delta t)$.

A quantitative analysis revealed that the deviations from prediction (68) of the rate equations are less than $3 \%$ of $b_{l 0}(0)$ if $\Delta \phi_{f} \geqslant 0.55 \pi, n_{l} \geqslant 10$, and $n_{l} n_{r} \geqslant 80$. Typical numbers that apply to the experiments in this work are $\Delta \nu_{\mathrm{rf}}$ $=750 \mathrm{~Hz}, \nu_{\mathrm{FM}}=100 \mathrm{~Hz}, \Delta \phi_{f}=2.2 \pi, n_{r}=1000$, and $n_{l}=20$. The conclusion is, therefore, that the interpretation of the NMR-ON relaxation curves in this work by the rate equations is justified.

Finally, it should be mentioned that, in order to confine the number of the parameters to a minimum, this analysis of the excitation process neglects several involvements: (i) The spin-lattice relaxation is completely neglected. (ii) A single resonance frequency for each spin is assumed. However, due to the small electric hyperfine interaction in cubic ferromagnets, the resonance is actually split into $2 I$ subresonances. (iii) $\phi_{f}$ is assumed to be the same for all spins. However, since the moment of a particular fast passage is not exactly the same for all spins, $\phi_{f}$ actually also varies from spin to spin, though much less than from passage to passage. (iv) The actual pattern of the modulation of the rf frequency may be more involved than a sawtooth modulation.

However, all those effects only further disturb the coherence between the rf field and the spins. The agreement with the rate equations should, therefore, be still better than demonstrated above.

\section{EXPERIMENTAL DETAILS}

The Fe sample was a circular single-crystal disk with (110) plane, $2.2 \mathrm{~mm}$ thick, and $12 \mathrm{~mm}$ in diameter. The purity of the sample and the flatness of the surface benefited from the fact that the sample was originally prepared for experiments on surface chemistry: For example, the bulk concentration of sulfur was reduced by baking at temperatures of 970-1120 K in flowing hydrogen for three weeks. The segregation of contaminants at the surface was reduced in an UHV chamber by hundred cycles of heating $(1000 \mathrm{~K}$, 10-30 min) and $\mathrm{Ar}^{+}$sputtering $\left(500 \mathrm{~K}, 750 \mathrm{eV}, 1 \mu \mathrm{A} \mathrm{cm}^{-2}\right.$, $30-10 \mathrm{~min})$. The final examination of the purity at the sur- 
face by Auger-electron and photoelectron spectroscopies revealed only about 0.4 at. \% phosphorus, 0.4 at. \% sulfur, 3 at. $\%$ carbon, and 1 at. \% oxygen.

$\mathrm{Hg}$ precursors of the probe nuclei ${ }^{186} \mathrm{Ir}$ and ${ }^{189} \mathrm{Ir}$ were coimplanted at the on-line mass separator ISOLDE at CERN (about $3 \times 10^{12}$ nuclei of ${ }^{186} \mathrm{Hg}$ and $8 \times 10^{12}$ of ${ }^{189} \mathrm{Hg}$, implantation voltage of $60 \mathrm{kV}$ ). To delimit the variation of position-dependent parameters such as the demagnetization field or the rf-field strength, the $\mathrm{Hg}$ beam was confined by a diaphragm to a spot of $4 \mathrm{~mm}$ diameter in the center of the disk. After the implantation, the sample was annealed for about $1 / 2 \mathrm{~h}$ at $970 \mathrm{~K}$ and slowly cooled down to room temperature.

The sample was then loaded into a ${ }^{3} \mathrm{He}-{ }^{4} \mathrm{He}$-dilution refrigerator and cooled down to temperatures in the $20 \mathrm{mK}$ range. The magnetic field was applied along the [100] direction in the sample plane. The orientation of the crystallographic axes relative to the magnetic field was accurate to $1^{\circ}$. The $\gamma$ anisotropy was measured by four Ge detectors, placed at $0^{\circ}, 90^{\circ}, 180^{\circ}$, and $270^{\circ}$ with respect to the direction of the magnetic field. The count rate ratio

$$
\epsilon=\frac{N\left(0^{\circ}\right)+N\left(180^{\circ}\right)}{N\left(90^{\circ}\right)+N\left(270^{\circ}\right)}-1
$$

was used to analyze the data. The temperature was primarily determined by a ${ }^{60} \mathrm{Co} \mathrm{Co}(\mathrm{hcp})$ nuclear orientation thermometer. However, because of the low sensitivity of that thermometer at temperatures above $20 \mathrm{mK}$, in most relaxation measurements, the temperature was determined via the equilibrium $\gamma$ anisotropy of ${ }^{186} \mathrm{Ir}$, which was calibrated with respect to the primary thermometer at lower temperatures.

The rf frequency was modulated in the following way: An external triangular FM was applied with bandwidth of $\pm 5 \mathrm{MHz}$ and frequency $\nu_{\mathrm{FM}}^{(1)}=100 \mathrm{~Hz}$. In addition, to reinforce the disturbance of the coherence between the rf field and the spins, a second internal triangular FM was applied with bandwidth of $\pm 200 \mathrm{~Hz}$ and frequency $\nu_{\mathrm{FM}}^{(2)}=1 \mathrm{~Hz}$. The half-width $\Delta \nu_{\mathrm{rf}}$ of the frequency fluctuations of the rf signal generator in the external modulation mode was about $750 \mathrm{~Hz}$. This was measured at nominally zero applied modulation voltage by a rf frequency analyzer.

The magnetic dipolar and the electric-quadrupolar parts of the relaxation were determined from the combined relaxation data on ${ }^{186} \mathrm{Ir}$ and ${ }^{189} \mathrm{Ir}$, as discussed in Ref. 15. In this work, only the magnetic relaxation constants of ${ }^{186} \mathrm{Ir}$ are quoted. Anyway, for that isotope, the quadrupolar contribution to the relaxation was only of the order of $1 \%$.

\section{MEASUREMENTS}

The static hyperfine interactions were determined by NMR-ON and modulated adiabatic fast passage on oriented nuclei (MAPON) ${ }^{50,51}$ Figure 3 shows the NMR-ON spectrum at $B_{\text {ext }}=0.1 \mathrm{~T}$. The magnetic resonance frequency and the subresonance separation were $\nu_{n}=794.68(20) \mathrm{MHz}$ and $\Delta \nu_{Q}=+0.838(2) \mathrm{MHz}$, respectively. Additional NMR-ON spectra were measured at $B_{\text {ext }}=0.5$ and $1.0 \mathrm{~T}$. From the field dependence of the resonance, $B_{\mathrm{dem}}^{(0)}=0.274(17) \mathrm{T}$ was de-

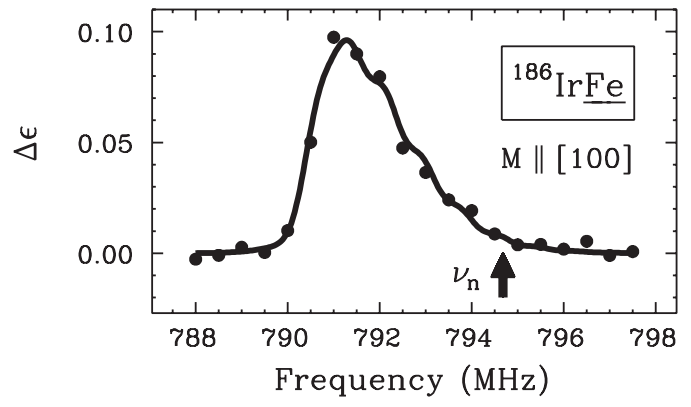

FIG. 3. NMR-ON spectrum of ${ }^{186} \mathrm{Ir}$ at $B_{\text {ext }}=0.1 \mathrm{~T} . \quad T$ $=46(2) \mathrm{mK}, \mathrm{FM}$ bandwidth $\pm 0.5 \mathrm{MHz}$. The interpretation of the only partly resolved subresonance structure made use of the knowledge of the distribution of $\Delta \nu_{Q}$ from the MAPON measurements.

duced. To excite all subresonances in the relaxation measurements, the frequency was modulated, for example, at $B_{\text {ext }}=0.1 \mathrm{~T}$ between 789.4 and $799.4 \mathrm{MHz}$.

The magnetization behavior was monitored via the $\gamma$ anisotropy of ${ }^{186} \mathrm{Ir}$, which remained constant at $95(1) \%$ of its saturation value for $B_{\text {ext }} \leqslant 0.25 \mathrm{~T}$, increased slightly between 0.25 and $0.40 \mathrm{~T}$, and remained at its saturation value for higher fields. This confirmed that the magnetization was essentially aligned along the [100] direction within the sample plane. Other alignments of the magnetization apparently only occurred at low fields in a small fraction of the sample. That fraction remained constant in the multidomain regime up to $B_{\mathrm{ext}}=B_{\mathrm{dem}}^{(0)}$ but disappeared at higher fields.

The angular-distribution coefficients of the most intense $\gamma$ transitions of ${ }^{186} \mathrm{Ir}$, which were needed for the description of the relaxation curves and for the thermometry via ${ }^{186} \mathrm{Ir}$, were determined by measurements of the $\gamma$ anisotropy as a function of the temperature between 10 and $23 \mathrm{mK}$. For example, $A_{2}=-0.311(2)$ and $A_{4}=-0.136(4)$ were obtained for the $297 \mathrm{keV}$ transition at $B_{\mathrm{ext}}=0.5 \mathrm{~T}$. (Here, $A_{i}$ corresponds to $A_{i} U_{i}$ in the notation of Ref. 43.)

The reliability of the relaxation measurement technique was tested by measurements at different rf-power levels. For example, at $B_{\text {ext }}=0.5 \mathrm{~T}$, the applied rf power $P_{\text {rf }}$ was varied in five measurements by a factor of 16 . Thereby the relative resonant reduction of the nuclear magnetization varied between $9 \%$ and $71 \%$, whereas the temperature varied between $k_{B} T / h \nu_{n}=0.60$ and $k_{B} T / h \nu_{n}=1.78$. Figure 4 shows three of the relaxation curves. All relaxation curves could perfectly be described by the theory. Moreover, the least squares fit results for $R, R_{\mathrm{rf}}$, and $f_{\mathrm{rf}}$ were all consistent, demonstrating the reliability of the measurement technique and of the interpretation of the relaxation curves. The fit results are shown in Fig. 5 as a function of $P_{\text {rf }}$.

Similarly consistent results were obtained at $B_{\text {ext }}=0.1 \mathrm{~T}$, where $P_{\mathrm{rf}}$ was varied in six measurements by a factor of 32 . The only deviation from the theory was that at the two highest rf-power levels, the increase of $R_{\mathrm{rf}}$ with $P_{\mathrm{rf}}$ was smaller than expected. However, at those high power levels, the time scale of the excitation by the rf field was extraordinarily short, of the order of 2-3 periods of the FM. It is not surprising that in this case, the picture of a continuous excitation process begins to fail.

Further tests examined the disturbance of the coherence of the rf field. According to Sec. IV, that disturbance manifests 


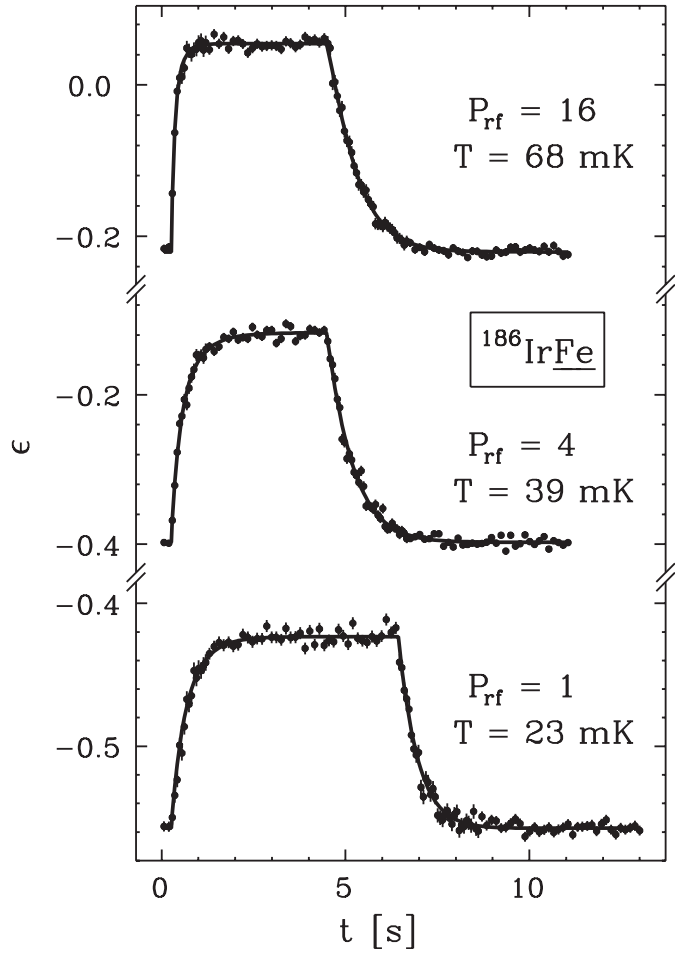

FIG. 4. ${ }^{186} \mathrm{IrFe}, B_{\text {ext }}=0.5 \mathrm{~T}$ : NMR-ON relaxation curves at different rf-power levels (and temperatures). $P_{\mathrm{rf}}$ in arbitrary units. The increase and the decrease of $\epsilon$ reflect the temporal evolution after switching the FM on and off, respectively.

itself in fluctuations of $\phi=\phi_{0}+\phi_{f}$, the relative phase of subsequent fast passages. As shown in Sec. IV, the fluctuations are expected to be large enough that the excitation of the spins can be described by rf transition rates as in the case of an incoherent irradiation. In this case, additional variations of $\phi$ should have no effect. This was confirmed in the following ways: (i) Relaxation measurements with and without the second FM, which varies $\phi_{0}$ periodically by more than

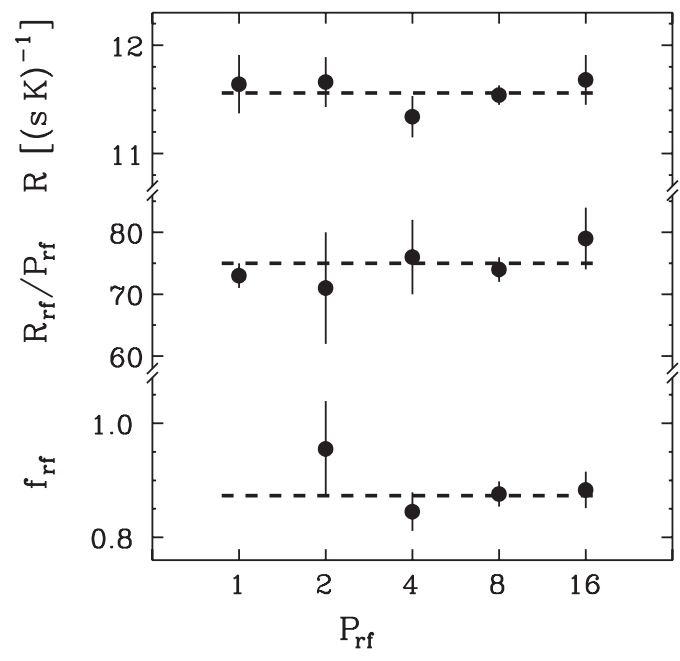

FIG. 5. ${ }^{186} \mathrm{IrFe}, B_{\mathrm{ext}}=0.5 \mathrm{~T}: R, R_{\mathrm{rf}} / P_{\mathrm{rf}}$, and $f_{\mathrm{rf}}$ from measurements at different rf-power levels. At the lowest power level, $f_{\mathrm{rf}}$ had to be taken from the other measurements, because $f_{\text {rf }}$ and $R_{\text {rf }}$ proved to be too correlated to determine both parameters independently.

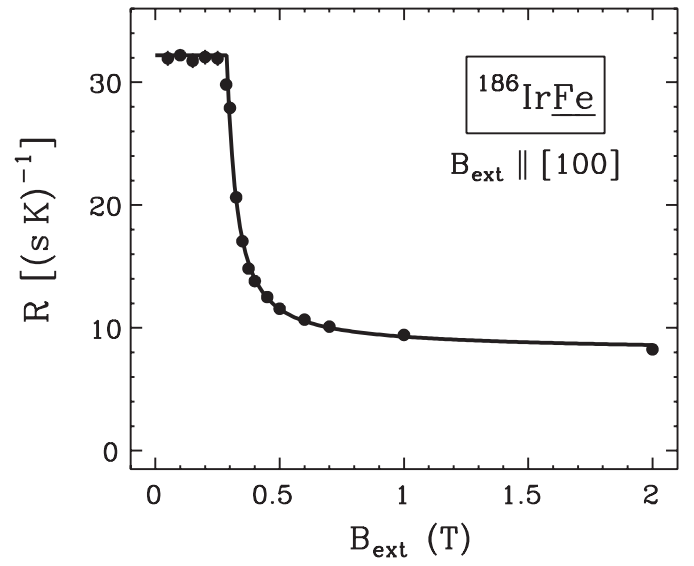

FIG. 6. ${ }^{186} \mathrm{IrFe}$ : Magnetic-field dependence of the nuclear spinlattice relaxation.

$2 \pi$, gave identical results. (ii) Applying only a single modulation, the resonance effect was measured as a function of $\nu_{\mathrm{FM}}$. In this way, $\Delta \phi_{f}$ was varied according to Eq. (67), but the resonance effect remained essentially constant between $\nu_{\mathrm{FM}}=50 \mathrm{~Hz}$ and $\nu_{\mathrm{FM}}=1 \mathrm{kHz}$.

Only for $\nu_{\mathrm{FM}}=5 \mathrm{kHz}$ and larger $\nu_{\mathrm{FM}}$ 's the resonance effect was significantly reduced as a result of the reduction of $\Delta \phi_{f}$. The resonance effect was also reduced for $\nu_{\mathrm{FM}}=20 \mathrm{~Hz}$ and smaller $\nu_{\mathrm{FM}}$ 's, because the time between the fast passages became of the order of the relaxation time. A similar dependence of the NMR-ON resonance effect on $\nu_{\mathrm{FM}}$ and a similar interpretation had already been reported in Ref. 41 for ${ }^{60} \mathrm{Co}$ in Fe.

The magnetic-field dependences of the spin-lattice relaxation and the enhancement factor were determined by relaxation measurements at 17 different fields between 0.05 and 2.0 T. $R$ and $R_{\mathrm{rf}} / P_{\mathrm{rf}}$, which is, apart from a prefactor, equivalent to $\eta^{2}$, are shown as a function of $B_{\text {ext }}$ in Figs. 6 and 7 , respectively. $f_{\mathrm{rf}}$ was in all cases consistent with the average value $f_{\text {rf }}=0.88(2)$.

The field dependence of $\eta$ was described by Eqs. (1) and (3), assuming $B_{a}=0.059 \mathrm{~T}$. This resulted in a perfect description of the field dependence of $R_{\mathrm{rf}} / P_{\mathrm{rf}}$ over 3 orders of magnitude. Only $B_{\mathrm{dem}}^{(0)}$ and the proportionality constant between $\eta^{2}$ and $R_{\mathrm{rf}} / P_{\mathrm{rf}}$ were adjusted via least squares fit. The solid

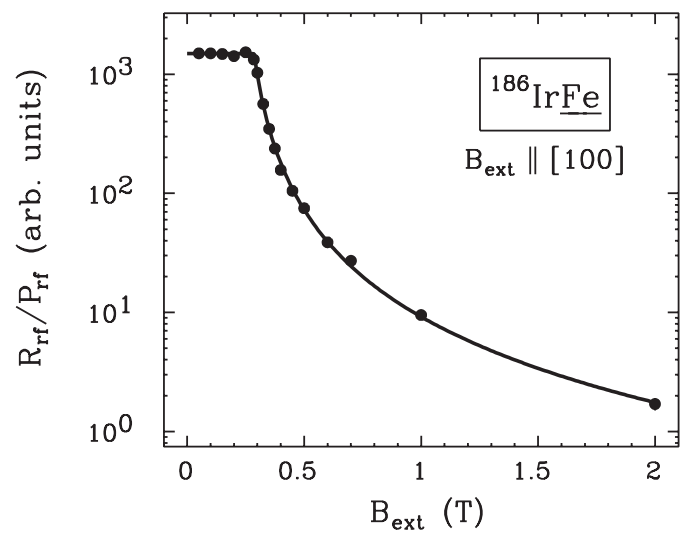

FIG. 7. ${ }^{186}$ IrFe: Magnetic-field dependence of the square of the enhancement factor. 
line in Fig. 7 shows the respective theoretical curve. If $B_{a}$ was also fitted to the data, $B_{a}=0.0607(11) \mathrm{T}$ was obtained, in agreement with the literature value.

$B_{\mathrm{dem}}^{(0)}=0.287(2) \mathrm{T}$ was obtained from the fit to the field dependence of $\eta^{2}$, in agreement with the value that was deduced from the field dependence of $\nu_{n}$, but in disagreement with the calculated value, $B_{\mathrm{dem}}^{(0)} \sim 0.20 \mathrm{~T}$. The deviation from the calculated value could not be resolved. In any case, the sharp bend of the field dependences at $B_{\text {ext }}=B_{\mathrm{dem}}^{(0)}$ showed that the assumption of a uniform value of $B_{\mathrm{dem}}^{(0)}$ for all probe nuclei was essentially justified.

The field dependence of $R$ could be described in different ways. The EFM in the form of Eq. (2) has the virtue that the magnitude and the form of the field dependence can be characterized by a simple expression without binding oneself to a particular explanation. The dashed line in Fig. 8 shows the fit of that model to the data. Using $B_{a}=0.059 \mathrm{~T}$, an almost perfect description was obtained with

$$
\begin{gathered}
R(0)=32.20(20)(\mathrm{s} \mathrm{K})^{-1}, \\
R(\infty)=8.69(22)(\mathrm{s} \mathrm{K})^{-1}, \\
\xi=1.39(4) .
\end{gathered}
$$

If, as it is done in this work, the field dependence of the relaxation is attributed to eddy-current and Gilbert dampings, $R$ is the sum of a field-independent part $R(\infty)$ and of the field-dependent contributions $R_{\text {ed }}$ and $R_{\mathrm{gi}}$. The data could perfectly be described in this way. The solid lines in the Figs. 6 and 8 show the respective theoretical curve. The following parameters were determined via least squares fit:

$$
\begin{gathered}
\sigma_{0} / \Lambda=7.1(6)(\mu \Omega \mathrm{cm} \mu \mathrm{m})^{-1}, \\
G=0.075(17) \mathrm{GHz}, \\
R(\infty)=7.4(5)(\mathrm{s} \mathrm{K})^{-1} .
\end{gathered}
$$

The quoted errors already take the uncertainty in $\sigma_{0}$ into account. $\sigma_{0}$ was assumed to be in the range $3.0-1000(\mu \Omega \mathrm{cm})^{-1}$, which corresponds to a residual resistivity ratio between 30 and $10^{4}$. The composition of the fielddependent part of the relaxation changes strongly with the field: At $B_{\text {ext }}=0 \mathrm{~T}$, for example, the quoted damping parameters imply $R_{\mathrm{ed}}=20.2(\mathrm{~s} \mathrm{~K})^{-1}$ and $R_{\mathrm{gi}}=4.6(\mathrm{~s} \mathrm{~K})^{-1}$, whereas at $B_{\text {ext }}=1 \mathrm{~T}$, for example, $R_{\text {ed }}=0.29(\mathrm{~s} \mathrm{~K})^{-1}$ and $R_{\mathrm{gi}}$ $=1.60(\mathrm{~s} \mathrm{~K})^{-1}$.

In the past, the field dependence of the relaxation had often been described by the EFM assuming $\xi=2$, and $B_{a}$ had been fitted to the data. To assess the results that had been obtained in this way, we also applied that traditional variant of the EFM. The following parameters were obtained via least squares fit:

$$
\begin{gathered}
B_{a}=0.091(4) \mathrm{T}, \\
R(\infty)=9.41(18)(\mathrm{s} \mathrm{K})^{-1} .
\end{gathered}
$$

The dotted line in Fig. 8 shows the respective theoretical curve. The field dependence of $R$ is remarkably well repro-

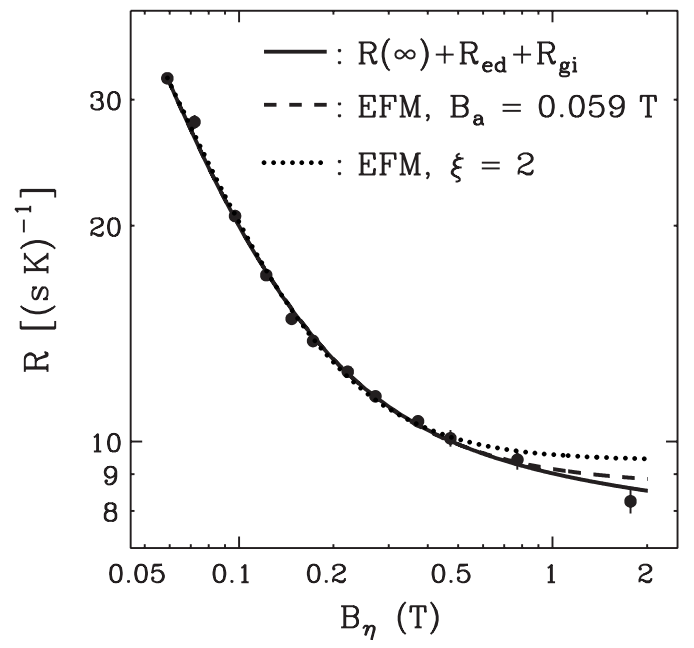

FIG. 8. ${ }^{186} \mathrm{IrFe}$ : Comparison of the field dependence of the spinlattice relaxation with the descriptions by $R=R(\infty)+R_{\mathrm{ed}}+R_{\mathrm{gi}}$ (solid line), by the EFM with $B_{a}=0.059 \mathrm{~T}$ and $\xi=1.39$ (dashed line), and by the EFM with $B_{a}=0.091 \mathrm{~T}$ and $\xi=2$ (dotted line). In order to show the low-field part more clearly, a double logarithmic scale was used, and $B_{\text {ext }}$ was converted into $B_{\eta}$, assuming $B_{\mathrm{dem}}^{(0)}=0.287 \mathrm{~T}$ and $B_{a}=0.059 \mathrm{~T}$.

duced, at least between 0 and $1 \mathrm{~T}$, although $B_{a}$ is clearly wrong.

\section{DISCUSSION}

\section{A. Origin of the magnetic-field dependence}

For all the relaxation mechanisms that were discussed in Sec. III, the magnetic-field dependence is actually a dependence on $B_{\eta}$. The knowledge of the magnetic-field dependence of $B_{\eta}$ is thus indispensable for the comparison between experiment and theory. Therefore, the experimental determination of the field dependence of $B_{\eta}$ in this work via the quantity $R_{\mathrm{rf}} / P_{\mathrm{rf}}$ was particularly important. It confirmed that $B_{\eta}$ was indeed given by Eq. (3) with $B_{a}=0.0607(11) \mathrm{T}$. This showed, in particular, that the value of $B_{a}$ and the magnetization behavior were not modified at the site of the probe nuclei by the presence of the impurity, by the closeness to the surface, or by other effects.

The discussion of the various potentially field-dependent relaxation mechanisms in Sec. III showed that most of them can be excluded as the source of the observed field dependence: The direct excitation of spin waves is not possible, since the spin-wave frequencies are larger than $\nu_{n}$. The excitation of sound waves is negligible. The field dependences of the various variants of the indirect spin-wave mechanism are given in Sec. III D. They are all too weak to explain the observed field dependence. A connection to domain walls can also be excluded, since the domain walls just disappear when the field dependence sets in at $B_{\mathrm{ext}}=B_{\mathrm{dem}}^{(0)}$.

In contrast, the relaxation via eddy-current and Gilbert dampings explains both the magnitude and the form of the field dependence. It provides a perfect description of the data. The used values of the damping parameters $\sigma_{0} / \Lambda$ and $G$ are well within the expected ranges, which were specified 
TABLE I. Parameters of the field dependence of the spin-lattice relaxation in Fe from different experiments in the [100] geometry. $\sigma_{0} / \Lambda$ was determined by a fit of $R(\infty)+R_{\mathrm{ed}}+R_{\mathrm{gi}}$, assuming a residual resistivity ratio between 30 and $10^{4}$. G $=0.075 \mathrm{GHz}$ was taken from the ${ }^{186} \mathrm{Ir}$ experiment, because the precision of the data was not sufficient for its determination in the other cases. $\xi, R(0)$, and $R(\infty)$ were determined by a fit of the EFM.

\begin{tabular}{ccccccc}
\hline \hline & $\begin{array}{c}\nu_{n} \\
\text { Isotope }\end{array}$ & Ref. & $\begin{array}{c}\sigma_{0} / \Lambda \\
{\left[(\mu \Omega \mathrm{cm} \mu \mathrm{m})^{-1}\right]}\end{array}$ & $\xi$ & $\begin{array}{c}{[R(0)-R(\infty)] / \nu_{n}^{2}} \\
{\left[(\mathrm{~s} \mathrm{~K})^{-1} \mathrm{GHz}^{-2}\right]}\end{array}$ & $\begin{array}{c}R(\infty) / \nu_{n}^{2} \\
{\left[(\mathrm{~s} \mathrm{~K})^{-1} \mathrm{GHz}^{-2}\right]}\end{array}$ \\
\hline${ }^{110 m} \mathrm{Ag}$ & 0.205 & 9 & $2.6(5)$ & $1.60(34)$ & $21.0(24)$ & $10.3(12)$ \\
${ }^{131} \mathrm{I}$ & 0.683 & 8 and 9 & $5.2(6)$ & $1.36(17)$ & $30.9(19)$ & $8.3(12)$ \\
${ }^{186} \mathrm{Ir}$ & 0.795 & This work & $7.1(6)$ & $1.39(4)$ & $37.2(5)$ & $13.8(3)$ \\
${ }^{191} \mathrm{Pt}$ & 0.320 & 52 & $2.7(3)$ & $1.13(30)$ & $21.9(20)$ & $9.9(14)$ \\
\hline \hline
\end{tabular}

in Secs. III E and III F. This strongly suggests that the relaxation via eddy-current and Gilbert dampings is indeed the source of the magnetic-field dependence of the nuclear spinlattice relaxation in Fe. Of course, further, hitherto unknown, sources cannot completely be excluded, since $\sigma_{0} / \Lambda$ and $G$ had to be adjusted via least squares fit. One should also be aware that the used values of $\sigma_{0} / \Lambda$ and $G$ may not represent the actual values of those damping parameters, because the surface effects were not taken into account.

Having identified the relaxation mechanism that is responsible for the magnetic-field dependence, we can now use the theory that has been worked out in Secs. III E and III F to establish the connection to previous theoretical work, to reinterpret the results of other experiments, to compare the field dependences in $\mathrm{Fe}, \mathrm{Co}$, and $\mathrm{Ni}$, and to judge the validity of the literature values of $R(\infty)$.

\section{B. Previous concepts}

The theory of the spin-lattice relaxation via eddy-current and Gilbert dampings contains several concepts that have already been suggested previously in the context with the field dependence of the relaxation in Fe: For example, the virtual excitation of spin waves, which was proposed together with the indirect spin-wave mechanism, is also one step in the relaxation via eddy-current and Gilbert damping. Moreover, the indirect spin-wave mechanism itself can be understood as one contribution to $R_{\mathrm{gi}}$.

It also turns out that the original concept of the EFM, the proportionality between the field-dependent part of the spinlattice relaxation and the square of the enhancement factor, indeed applies in some sense to the relaxation via the virtual excitation of spin waves. However, it is the enhancement factor

$$
\eta(q)=\frac{B_{\mathrm{HF}}}{B_{\eta}+\left(D q^{2}\right) /\left(\hbar\left|\gamma_{e}\right|\right)}
$$

of transversal fields with wave vector $\mathbf{q}$ that enters quadratically via the factor $\left[\omega_{x}^{(0)}\right]^{-2}$ in Eq. (28). The field dependence thus essentially combines $[\eta(q)]^{2}$ terms from all momentum transfers, whereas within the EFM, it is approximated by a $\eta^{\xi}$ term, where $\eta$ is the $q=0$ limit of $\eta(q)$. Since the field dependence of $\eta(q)$ decreases with increasing $q, \xi$ is smaller than 2. To which extent depends on the weighting of the individual momentum transfers.
This relationship to the EFM also reveals that the form of the field dependence is indeed a signature of the proposed relaxation mechanism: It was first a puzzle that the fielddependent part of the relaxation seemed to be proportional to $\eta^{2}$, as if only $q=0$ would contribute. That puzzle is now solved: On one hand, our experiment shows that $\xi$ is indeed smaller than 2. On the other hand, the eddy-current damping implies a particularly strong weighting of small momentum transfers: $\operatorname{Im}\left[\omega_{x}^{(\mathrm{ed})}\right]$ and $\operatorname{Im}\left[\omega_{y}^{(\mathrm{ed})}\right]$ are proportional to $q^{-3}$ for $q \delta \gg 1$ and $q \Lambda \gg 1$. Such a strong preference of small $q$ 's is required to explain the strong field dependence with $\xi$ close to 1.4. Moreover, it is not readily reproduced by other relaxation mechanisms, as the discussion in Sec. III D showed: All discussed variants of the indirect spin-wave mechanism have distinctly weaker field dependences, because the small momentum transfers are less strongly weighted.

\section{Other experiments}

A major problem of the interpretation of previous experiments is the fact that the field dependence of $B_{\eta}$ is not well known in most cases. $B_{\eta}$ is reasonably well known only for the experiments on Fe single crystals where the magnetic field was applied along the [100] direction in the sample plane. The data of those experiments were redescribed by both $R(\infty)+R_{\text {ed }}+R_{\text {gi }}$ and the EFM. $B_{\eta}$ was assumed to be given by Eq. (3) with $B_{a}=0.059$ T. $B_{\mathrm{dem}}^{(0)}$ was fitted to the data. Table I summarizes the results.

The form of the field dependences supports the interpretation by $R_{\mathrm{ed}}+R_{\mathrm{gi}}$. The parameter $\xi$ is a measure of the respective agreement with the theory. It is, within the error, in all cases close to 1.4 , as expected for $R_{\text {ed }}+R_{\text {gi }}$. In contrast, the magnitudes of the field dependences are inconsistent in so far as they cannot be described by the same set of damping parameters $\sigma_{0} / \Lambda$ and $G$. This also manifests itself by the differences in $[R(0)-R(\infty)] / \nu_{n}^{2}$, which cannot be explained by the weak $\nu_{n}$ dependence of $\left(R_{\mathrm{ed}}+R_{\mathrm{gi}}\right) / \nu_{n}^{2}$. This inconsistency may be attributed to surface effects. This would imply that differences in the surface preparation or in the location of the probe nuclei had changed the field-dependent part of the relaxation by up to a factor of 2 .

Relaxation measurements on Fe single crystals were also performed with the magnetic field applied along other 
crystallographic directions. ${ }^{8,9,14}$ In most cases, geometries were investigated where $B_{\eta}$ vanishes, at least nominally, for certain values of the magnetic field. Those experiments nicely demonstrated that it is indeed the quantity $B_{\eta}$ that has to become small to obtain a large relaxation rate. However, quantitative conclusions are not possible, because the field dependence of $B_{\eta}$ was not determined experimentally, nor can it reliably be calculated. The problem with the calculation is that, when $B_{\eta}$ becomes very small, the magnetization behavior becomes extremely sensitive to misalignments and inhomogeneities, which are always unavoidable to some extent.

Most of the previous relaxation experiments were performed on cold-rolled polycrystalline foils of dilute $\mathrm{Fe}$ alloys, where a well-founded description of the field dependence of $B_{\eta}$ is not feasible. Nevertheless, the previous interpretation of those experiments is of interest, because it seemed to support the assumption $\xi=2$ : The field dependence of the relaxation could be described by the EFM and $\xi=2$, if $B_{\eta}$ was parametrized by $B_{a}+B_{\mathrm{ext}}$, and $B_{a}$ was adjusted via least squares fit. ${ }^{6,7,53,54}$ However, $B_{a}$ and $\xi$ are strongly correlated, and over a fairly large magnetic-field region, a wrong choice of one of those parameters can be compensated by a wrong choice of the other parameter. This was demonstrated in this work for ${ }^{186} \mathrm{IrFe}$ : The field dependence of $R$ could almost equally well be described by $\xi$ $=1.39$ and the "correct" $B_{a}$ or by $\xi=2$ and a "wrong," distinctly larger $B_{a}$. Therefore, the values of $\xi$ and $B_{a}$ from the previous interpretation of those experiments are meaningless.

The theory of $R_{\text {ed }}+R_{\mathrm{gi}}$ is also important for the understanding of experiments where the relaxation rates of different isotopes of the same element in the same sample are compared, because it predicts deviations from the usually expected proportionality to $\nu_{n}^{2}$, which is well established, for example, for $R(\infty) .{ }^{53} \mathrm{An}$ experiment of this kind on ${ }^{60} \mathrm{Co}$ $\left(\nu_{n}=0.166 \mathrm{GHz}\right)$ and ${ }^{58} \mathrm{Co}\left(\nu_{n}=0.442 \mathrm{GHz}\right)$ in Fe was reported in Ref. 14. From the low-field measurements of that work,

$$
\frac{\left.\left(R^{\prime} / \nu_{n}^{2}\right){ }^{58} \mathrm{Co}\right)}{\left(R^{\prime} / \nu_{n}^{2}\right)\left({ }^{60} \mathrm{Co}\right)}=0.70(12)
$$

can be deduced, where $R^{\prime}$ denotes the field-dependent part of $R$. $B_{\eta}$ was presumably in the range $0.02-0.06 \mathrm{~T}$. The deviation from $R^{\prime} \propto \nu_{n}^{2}$ is, at least in part, explained by the theory of $R_{\text {ed }}+R_{\text {gi }}$, according to which

$$
\frac{\left.\left[\left(R_{\mathrm{ed}}+R_{\mathrm{gi}}\right) / \nu_{n}^{2}\right]{ }^{58} \mathrm{Co}\right)}{\left.\left[\left(R_{\mathrm{ed}}+R_{\mathrm{gi}}\right) / \nu_{n}^{2}\right]{ }^{60} \mathrm{Co}\right)}=0.81-0.89,
$$

if the damping parameters are similar to those of the ${ }^{186} \mathrm{IrFe}$ experiment. Unfortunately, the statistical significance of the quoted data is poor and technical details of the respective measurements have been questioned. ${ }^{7}$

Relaxation measurements on different isotopes of the same element in the same sample were also reported in Refs. 15 and 42. They were used to deduce the electricquadrupolar part of the spin-lattice relaxation, which is possible, if the quadrupole moment of one of the isotopes is sufficiently large. However, to separate the magnetic-dipolar and the electric-quadrupolar parts of the relaxation, a scaling of the magnetic part with $\nu_{n}^{2}$ was assumed. Therefore, the deduced field dependences of the quadrupolar relaxation are invalid. In contrast, the deduced high-field limits should essentially be correct.

In the case of the measurements of this work on ${ }^{186} \mathrm{Ir}$ and ${ }^{189} \mathrm{Ir}$, the data were reanalyzed, taking the $\nu_{n}$ dependences of $R_{\mathrm{ed}}$ and $R_{\mathrm{gi}}$ into account. The revised result for the ratio of the low-field and the high-field quadrupolar relaxation constants is $R_{q}(0 \mathrm{~T}) / R_{q}(2 \mathrm{~T})=0.97(15)$. Thus, there is no significant field dependence of the quadrupolar relaxation, in contrast to our previous conclusion in Ref. 15.

This field independence of the quadrupolar relaxation is in accord with the theory. Indeed, $R_{\mathrm{ed}}$ and $R_{\mathrm{gi}}$ also contribute to the quadrupolar relaxation, because the magnetization is also coupled to the nuclear quadrupole moment via the spin-orbitinduced electric-field gradient, but the contributions are too small to be observable. The form of these contributions is well known from the similar but much stronger contribution of the indirect spin-wave mechanism to the quadrupolar relaxation in the rare earth metals: ${ }^{30}$ The net effect is that $R_{\text {ed }}$ and $R_{\mathrm{gi}}$ must be calculated for each transition probability $W_{m+1, m}$ separately with $\nu_{n}$ replaced by the respective transition frequency $\nu_{m+1, m}$ of the quadrupolar-split resonance spectrum. In Fe, the effect is negligible, because the $\nu_{m+1, m}$ 's differ only slightly from $\nu_{n}$.

\section{Magnetic-field dependence in $\mathrm{Co}$ and $\mathrm{Ni}$}

Distinct magnetic-field dependences of the nuclear spinlattice relaxation have also been observed in $\mathrm{Co}(\mathrm{hcp}){ }^{55}$ $\mathrm{Co}(\mathrm{fcc}),{ }^{56}$ and $\mathrm{Ni} .{ }^{9}$ A detailed comparison with the theory is not possible, because the field dependence of $B_{\eta}$ is not sufficiently well known for those experiments. However, estimates of the typical magnitudes of $R_{\mathrm{ed}}, R_{\mathrm{gi}}$, and $R(\infty)$ can show at least whether major differences to the situation in $\mathrm{Fe}$ are to be expected. To estimate $R_{\text {ed }}$ and $R_{\mathrm{gi}}$ in Co and Ni, $D$ and $G$ were taken from Refs. 36 and 57-59. As discussed below, in the case of $G$, the room-temperature value should be used, which is, for $\mathrm{Co}$ and $\mathrm{Ni}$, distinctly smaller than the low-temperature value. $\sigma_{0} / \Lambda$ should be of the same order of magnitude in $\mathrm{Fe}, \mathrm{Co}$, and $\mathrm{Ni}$. Data on $R(\infty)$ in $\mathrm{Fe}, \mathrm{Co}$, and $\mathrm{Ni}$ are available, for example, from Refs. 3, 9, 53, 55, and $60-63$.

In the case of Co as the host, $R_{\mathrm{ed}}, R_{\mathrm{gi}}$, and $R(\infty)$ turn out to be of the same order of magnitude as in Fe. Thus, decisive differences to the situation in $\mathrm{Fe}$ are not expected, apart from differences in the field dependence of $B_{\eta}$.

In contrast, for $\mathrm{Ni}$ as the host, $R_{\mathrm{ed}}$ is of the same order of magnitude as in Fe, but $R_{\mathrm{gi}}$ is larger by a factor of 20 and $R(\infty)$ by typically 1 order of magnitude, if the comparison is made for the same values of $\nu_{n}$ and $B_{\eta}$. Thus, if $B_{\eta}$ is of the order of $0.1 \mathrm{~T}$, the relaxation is faster than in the high-field limit by factors that are similar to those in Fe. However, in $\mathrm{Ni}$, this is largely due to $R_{\mathrm{gi}}$ and not to $R_{\text {ed }}$. This has the following consequences: The field dependence of the relaxation is much weaker than in $\mathrm{Fe}$, with $\xi$ close to 0.4. More- 
over, this implies that even at relatively large fields of the order of $1 \mathrm{~T}$, a large fraction of the relaxation may be due to the field-dependent part.

It was also calculated which value of $R_{\mathrm{gi}}$ in $\mathrm{Ni}$ is expected if the low-temperature value of $G$ is used in combination with Eq. (57). It turned out that the use of the lowtemperature value is in contradiction to the experiment: For ${ }^{110 m} \mathrm{Ag}$ in $\mathrm{Ni}$ at $B_{\mathrm{ext}}=0$, for example, in this way, $R_{\mathrm{gi}}$ $=1.1(\mathrm{~s} \mathrm{~K})^{-1}$ is predicted, which is much larger than the observed relaxation constant of about $R \sim 0.2(\mathrm{~s} \mathrm{~K})^{-1}$ in this case. $^{9}$ In contrast, if the room-temperature value of $G$ is used, $R_{\mathrm{gi}}=0.19(\mathrm{~s} \mathrm{~K})^{-1}$ is predicted, which is of the right order of magnitude. This finding confirms the following interpretation of the temperature dependence of $G$ : According to Refs. 58 and $64, G$ is the sum of a largely temperatureindependent contribution and of a low-temperature contribution, which is negligible for $T>150 \mathrm{~K}$ and which shows similar temperature and wave-vector dependences as the conductivity. The wave-vector dependence implies that the respective contribution to $R_{\mathrm{gi}}$ is several orders of magnitude smaller than suggested by the literature values of $G$, because the wave vectors that are relevant for $R_{\mathrm{gi}}$ are much larger than those that are relevant for the ferromagnetic-resonance experiments that are used to determine $G$. The consequence is that the low-temperature contribution to $G$ makes only a negligible contribution to the spin-lattice relaxation.

\section{E. High-field limits}

The high-field limits of the spin-lattice relaxation are important for the comparison with the ab initio calculations, because the available calculations only take account of essentially field-independent contributions. Most literature values of $R(\infty)$ in $\mathrm{Fe}$ were deduced by the EFM assuming $\xi=2 .{ }^{53}$ If the data of this work are interpreted in this way, one obtains $R(\infty)=9.41(18)(\mathrm{K} \mathrm{K})^{-1}$. This is close to $R(\infty)$ $=8.97(25)(\mathrm{s} \mathrm{K})^{-1}$, which follows from the literature value for IrFe from Ref. 53, if that value is corrected for the quadrupolar contribution to the relaxation ${ }^{42}$ and if a consistent set of nuclear moments is used to convert that value to ${ }^{186} \mathrm{Ir}$.

In contrast, if the data are interpreted by $R(\infty)+R_{\mathrm{ed}}+R_{\mathrm{gi}}$, the parameter $R(\infty)$ is about $20 \%$ smaller. This suggests that the actual high-field limits are smaller than the literature values by amounts of the order of $20 \%$.

\section{ACKNOWLEDGMENTS}

We appreciate very much the effort which was put by the Orsay group into the development of the liquid $\mathrm{Pb}$ target at ISOLDE. We also wish to thank E. Smolic for experimental help and H. D. Rüter for communication of unpublished work.
${ }^{1}$ M. Kontani, T. Hioki, and Y. Masuda, J. Phys. Soc. Jpn. 32, 416 (1972).

${ }^{2}$ E. Klein, Hyperfine Interact. 15/16, 557 (1983).

${ }^{3}$ E. Klein, in Low-Temperature Nuclear Orientation, edited by N. J. Stone and H. Postma (North-Holland, Amsterdam, 1986), Chap. 12.

${ }^{4}$ T. Moriya, J. Phys. Soc. Jpn. 18, 516 (1963).

${ }^{5}$ T. Hioki and Y. Masuda, J. Phys. Soc. Jpn. 43, 1200 (1977).

${ }^{6}$ M. Kopp and E. Klein, Hyperfine Interact. 11, 153 (1981).

${ }^{7}$ C. Bobek, R. Dullenbacher, and E. Klein, Hyperfine Interact. 77, 327 (1993).

${ }^{8}$ H. D. Rüter, W. Haaks, E. W. Duczynski, E. Gerdau, D. Visser, and L. Niesen, Hyperfine Interact. 9, 385 (1981).

${ }^{9}$ E. W. Duczynski, Ph.D. thesis, Universität Hamburg, 1983.

${ }^{10}$ V. Jaccarino, N. Kaplan, R. E. Walstedt, and J. H. Wernick, Phys. Lett. 23, 514 (1966).

${ }^{11}$ F. Hartmann-Boutron and D. Spanjaard, J. Phys. (Paris) 34, 217 (1973).

${ }^{12}$ B. G. Turrell, Hyperfine Interact. 7, 429 (1980).

${ }^{13}$ D. Visser, Ph.D. thesis, Rijksuniversiteit Groningen, 1981.

${ }^{14}$ W. van Rijswijk, H. S. van der Rande, A. A. Jilderda, and W. J. Huiskamp, Hyperfine Interact. 39, 23 (1988).

${ }^{15}$ G. Seewald, E. Zech, H. J. Körner, D. Borgmann, M. Dietrich, and ISOLDE Collaboration, Phys. Rev. Lett. 88, 057601 (2002).

${ }^{16}$ A. M. Portis and A. C. Gossard, J. Appl. Phys. 31, 205S (1960).

${ }^{17}$ J. Smit and H. G. Beljers, Philips Res. Rep. 10, 113 (1955).

${ }^{18}$ J. Korringa, Physica (Amsterdam) 16, 601 (1950).

${ }^{19}$ Y. Obata, J. Phys. Soc. Jpn. 18, 1020 (1963).

${ }^{20} \mathrm{~J}$. Winter, Magnetic Resonance in Metals (Oxford University
Press, Oxford, 1970), Appendix 1.

${ }^{21}$ M. Weger, Phys. Rev. 128, 1505 (1962).

${ }^{22}$ A. Honma, Phys. Rev. 142, 306 (1966).

${ }^{23}$ M. B. Stearns, in Magnetic Properties of Metals, edited by H. P. Wijn, Landolt-Börnstein, New Series, Group III, Vol. 19, pt. A (Springer, Berlin, 1986).

${ }^{24}$ C. Herring and C. Kittel, Phys. Rev. 81, 869 (1951).

${ }^{25}$ L. R. Walker, in Magnetism, edited by G. T. Rado and H. Suhl (Academic, New York, 1963), Vol. I, Chap. 8.

${ }^{26}$ T. G. Phillips and H. M. Rosenberg, Rep. Prog. Phys. 29, 285 (1966).

${ }^{27}$ G. E. H. Reuter and E. H. Sondheimer, Proc. R. Soc. London, Ser. A 195, 336 (1948).

${ }^{28}$ D. C. Mattis and G. Dresselhaus, Phys. Rev. 111, 403 (1958).

${ }^{29}$ P. Pincus and J. Winter, Phys. Rev. Lett. 7, 269 (1961).

${ }^{30}$ N. Sano, S. Kobayashi, and J. Itoh, Suppl. Prog. Theor. Phys. 46, 84 (1970).

${ }^{31}$ Y. Masuda, T. Hioki, and M. Kontani, Int. J. Magn. 6, 143 (1974).

${ }^{32}$ H. Akai, Hyperfine Interact. 43, 255 (1988).

${ }^{33}$ J. Callaway and C. S. Wang, Phys. Rev. B 16, 2095 (1977).

${ }^{34}$ R. G. Chambers, Proc. R. Soc. London, Ser. A 215, 481 (1952).

${ }^{35} \mathrm{~J}$. Bass, in Metals: Electronic Transport Phenomena, edited by K.-H. Hellwege and J. L. Olsen, Landolt-Börnstein, New Series, Group III, Vol. 15, pt. A (Springer, Berlin, 1982), Chap. 1, Sec. 2.

${ }^{36}$ F. Schreiber, J. Pflaum, Z. Frait, T. Mühge, and J. Pelzl, Solid State Commun. 93, 965 (1995).

${ }^{37}$ J. Dho and S. Lee, Phys. Rev. B 56, 7835 (1997). 
${ }^{38}$ H. Enokiya, J. Phys. Soc. Jpn. 42, 796 (1977).

${ }^{39}$ J. M. Winter, Phys. Rev. 124, 452 (1961).

${ }^{40}$ E. Matthias and R. J. Holliday, Phys. Rev. Lett. 17, 897 (1966).

${ }^{41}$ F. Bacon, J. A. Barclay, W. D. Brewer, D. A. Shirley, and J. E. Templeton, Phys. Rev. B 5, 2397 (1972).

${ }^{42}$ G. Seewald, E. Zech, and H.-J. Körner, Phys. Rev. B 70, 064419 (2004).

${ }^{43}$ K. S. Krane in Low-Temperature Nuclear Orientation, edited by N. J. Stone and H. Postma (North-Holland, Amsterdam, 1986), Chap. 2.

${ }^{44}$ E. Hagn and E. Zech, Phys. Lett. 101A, 49 (1984).

${ }^{45}$ N. J. Stone, in Low-Temperature Nuclear Orientation, edited by N. J. Stone and H. Postma (North-Holland, Amsterdam, 1986), Chap. 13.

${ }^{46}$ R. G. Ernst, G. Bodenhausen, and A. Wokaun, Principles of Nuclear Magnetic Resonance in One and Two Dimensions (Clarendon, Oxford, 1987), Chap. 2, Sec. 1.

${ }^{47}$ R. M. Steffen and K. Alder in The Electromagnetic Interaction in Nuclear Spectroscopy, edited by W. D. Hamilton (NorthHolland, Amsterdam, 1975), Chap. 12.

${ }^{48}$ D. M. Brink and C. R. Satchler, Angular Momentum (Clarendon, Oxford, 1962).

${ }^{49}$ A. Abragam, The Principles of Nuclear Magnetism (Clarendon, Oxford, 1961), pp. 424-433.

${ }^{50}$ P. T. Callaghan, P. J. Back, and D. H. Chaplin, Phys. Rev. B 37, 4900 (1988).

${ }^{51}$ P. J. Back, D. H. Chaplin, and P. T. Callaghan, Phys. Rev. B 37,
4911 (1988).

${ }^{52}$ G. Seewald, E. Hagn, E. Zech, R. Kleyna, M. Voß, D. ForkelWirth, and A. Burchard (unpublished).

${ }^{53}$ T. Funk, E. Beck, W. D. Brewer, C. Bobek, and E. Klein, J. Magn. Magn. Mater. 195, 406 (1999).

${ }^{54}$ M. Kopp, B. Kazemi-Far, and E. Klein, Z. Phys. B: Condens. Matter 44, 73 (1981).

${ }^{55}$ G. Seewald, E. Zech, H. Ratai, R. Schmid, R. Stadler, O. Schramm, C. König, B. Hinfurtner, E. Hagn, M. Deicher, R. Eder, and D. Forkel-Wirth, Hyperfine Interact. 155, 77 (2004).

${ }^{56}$ J. Herker, Diploma thesis, TU München, 1994.

${ }^{57}$ K. Hüller, J. Magn. Magn. Mater. 61, 347 (1986).

${ }^{58}$ S. M. Bhagat and P. Lubitz, Phys. Rev. B 10, 179 (1974).

${ }^{59}$ G. Dewar, B. Heinrich, and J. F. Cochran, Can. J. Phys. 55, 821 (1977).

${ }^{60}$ M. Matsumura, T. Kohara, and K. Asayama, J. Phys. Soc. Jpn. 49, 2179 (1980).

${ }^{61}$ R. L. Streever and P. J. Caplan, Phys. Lett. 38A, 439 (1972).

${ }^{62}$ T. Ohtsubo, D. J. Cho, Y. Yanagihashi, K. Komatsuzaki, K. Mizushima, S. Muto, and S. Ohya, in Hyperfine Interact. C, edited by M. Rots, A. Vantomme, J. Dekoster, R. Coussement, and G. Langouche (J. C. Baltzer AG, Basel, 1996), p. 577.

${ }^{63}$ G. Seewald, B. Hinfurtner, E. Zech, E. Hagn, A. Burchard, D. Forkel-Wirth, R. Eder, and ISOLDE Collaboration, Eur. Phys. J. B 35, 449 (2003).

${ }^{64}$ V. Korenman and R. E. Prange, Phys. Rev. B 6, 2769 (1972). 